\title{
Novel Mechanistic Insight into the Molecular Basis of Amyloid Polymorphism and Secondary Nucleation during Amyloid Formation
}

\author{
Jae Sun Jeong ${ }^{1}$, Annalisa Ansaloni ${ }^{2}$, Raffaele Mezzenga $^{3}$, Hilal A. Lashuel ${ }^{2}$ and Giovanni Dietler ${ }^{1}$
}

1 - Laboratory of Physics of Living Matter, École Polytechnique Fédérale de Lausanne, CH-1015 Lausanne, Switzerland

2 - Laboratory of Molecular and Chemical Biology of Neurodegeneration, École Polytechnique Fédérale de Lausanne,

$\mathrm{CH}-1015$ Lausanne, Switzerland

3 - Laboratory of Food and Soft Materials Science, Institute of Food, Nutrition and Health, Eidgenössische Technische Hochschule Zürich, Schmelzbergstrasse 9, LFO E23, 8092 Zürich, Switzerland

Correspondence to Hilal A. Lashuel and Giovanni Dietler: hilal.lashuel@epfl.ch; giovanni.dietler@epfl.ch http://dx.doi.org/10.1016/j.jmb.2013.02.005

Edited by S. Radford

\begin{abstract}
The formation of amyloid $\beta(A \beta)$ fibrils is crucial in initiating the cascade of pathological events that culminates in Alzheimer's disease. In this study, we investigated the mechanism of $A \beta$ fibril formation from hydrodynamically well defined species under controlled aggregation conditions. We present a detailed mechanistic model that furnishes a novel insight into the process of $A \beta 42$ fibril formation and the molecular basis for the different structural transitions in the amyloid pathway. Our data reveal the structure and polymorphism of $A \beta$ fibrils to be critically influenced by the oligomeric state of the starting materials, the ratio of monomeric-to-aggregated forms of $A \beta 42$ (oligomers and protofibrils), and the occurrence of secondary nucleation. We demonstrate that monomeric $A \beta 42$ plays an important role in mediating structural transitions in the amyloid pathway, and for the first time, we provide evidences that $A \beta 42$ fibrillization occurs via a combined mechanism of nucleated polymerization and secondary nucleation. These findings will have significant implications to our understanding of the molecular basis of amyloid formation in vivo, of the heterogeneity of $A \beta$ pathology (e.g., diffuse versus amyloid plaques), and of the structural basis of $A \beta$ toxicity.
\end{abstract}

(C) 2013 Elsevier Ltd. All rights reserved.

\section{Introduction}

Plaques resulting from the aggregation and accumulation of fibrillar amyloid $\beta(A \beta)$ peptides in the brain are a hallmark of Alzheimer's disease. ${ }^{1,2}$ $A \beta$ peptides evolve from the sequential cleavage of amyloid precursor proteins by $\beta$ - and $\gamma$ secretases. ${ }^{3,4}$ The predominant peptide forms are $A \beta 40$ and $A \beta 42$. Although the levels of $A \beta 40$ peptides are significantly higher than those of $A \beta 42$, the latter form is more toxic, has a much higher propensity to aggregate, and is believed to be crucial in initiating amyloid formation and the pathogenesis of Alzheimer's disease. ${ }^{5}$ The insoluble plaques that are formed in patients with Alzheimer's disease are composed of $A \beta$ fibrils. An increase in the production of $A \beta$ peptides and/or fibril formation could accelerate the onset of the disease. Hence, therapeutic strategies focus on lowering the levels of $A \beta$ peptides and/or inhibiting their aggregation.

In vitro, synthetic and recombinant forms of $A \beta$ peptides undergo structural changes leading to the formation of oligomeric intermediates and amyloid fibrils that resemble those isolated from the brains of patients with Alzheimer's disease. ${ }^{6-8}$ Biophysical studies relating to the aggregation of $A \beta$ peptides and to fibrillogenesis have improved our understanding of the mechanism underlying amyloid formation in vivo and have formed the basis for the hypothesis that this process is implicated in the pathogenesis of Alzheimer's disease. Amyloid formation comprises a highly ordered process of nucleated polymerization, which is characterized by a slow nucleation phase during which $A \beta$ monomers aggregated to form 
oligomeric nuclei, and a rapid growth phase involving the addition of monomers, the formation of fibrils, and their growth. ${ }^{9,10}$ Previous studies involving in situ atomic force microscopy (AFM) or transmission electron microscopy (TEM) have revealed $A \beta 42$ fibrillogenesis to proceed via the formation of highmolecular-weight protofibrils. ${ }^{11,12}$ The kinetics of protofibril formation and stability and the structure of the final fibrils depend greatly upon ambient conditions such as $\mathrm{pH}$, temperature, buffer composition, and peptide concentration, ${ }^{13-20}$ as well as upon the hydrophobicity or hydrophilicity of the substrate. ${ }^{21}$ Although the mechanism underlying the aggregation of $A \beta$ peptides in vitro has been the subject of numerous investigations over the past two decades, many fundamental questions remain unanswered. For example, (1) what triggers the aggregation of $A \beta$ peptides? (2) What is the relationship between the different oligomeric intermediates in the amyloid pathway? (3) What are the molecular and structural factors that determine the final structure and morphology of the $A \beta$ fibrils? To address these and other issues, we carried out a series of AFM studies-which included real-time measurements - to define the mechanisms underlying oligomerization, the formation of protofibrils, and the formation, association, and growth of fibrils under controlled conditions using $A \beta$ species of welldefined size and morphology. Motivated by initial data from our laboratory - which have suggested the existence of secondary-nucleation sites on the surfaces of preformed A 342 fibrils-we extended our studies to determine the structural basis of secondary nucleation and its role in determining the final structure and morphology of $A \beta$ fibrils.

Our findings afford a novel insight into the mechanism of $A \beta 42$ fibril formation and growth, as well as into the molecular basis of polymorphism, and for the first time, we provide evidences for the existence of secondary-nucleation sites on the surfaces of $A \beta 42$ fibrils. Furthermore, these secondary-nucleation sites are not a generic property of amyloid fibrils per se but specific to the type-2 variety. In addition, we demonstrate that the concentration of A 442 monomers at different stages of the amyloid pathway is crucial in determining the structural properties and heterogeneity of the fibrils formed.

\section{Results}

\section{Amyloid formation from monomeric A $\beta 42$ (Aß42-M)}

To elucidate the mechanism of amyloid formation from $A \beta 42$, we employed AFM to monitor the aggregation kinetics, as well as to characterize the structural properties and the heterogeneity of the oligomers and fibrils formed from pure preparations of monomeric $A \beta 42$ (AB42-M). After an incubation period of $30 \mathrm{~min}$ at $37^{\circ} \mathrm{C}$, spherical oligomers $(1.00 \pm 0.30 \mathrm{~nm}$ in diameter) and short protofibrils (arrows in Fig. 1a) were detected, which is consistent with previous observations. ${ }^{22}$ Within $24 \mathrm{~h}$, most of the spherical oligomers had disappeared and been replaced by short, flexible chain-like protofibrils (Fig. 1b), with an average cross-sectional diameter of $3.95 \pm 0.64 \mathrm{~nm}$ and an average length of $115.42 \pm$ $25.84 \mathrm{~nm}$. This finding is likewise consistent with previous observations. ${ }^{23}$ Within the protofibrils, the spacing between the individual spherical oligomers, which are 3-4 nm in height, ranged between 10 and $25 \mathrm{~nm}$. The height of these oligomers is identical with that of the spherical oligomers that form at $24 \mathrm{~h}$ prior or coincident with protofibril formation, suggesting that these beaded chain-like oligomer via linear growth without changing their height. Some of protofibrils were straight (arrows in Fig. 1b) and appeared preferentially to form fibrils with a periodicity of either $40.48 \pm 8.83 \mathrm{~nm}$ or $26.47 \pm 5.69 \mathrm{~nm}$ (Fig. 1b, inset). After an incubation period of $48 \mathrm{~h}$, the spherical oligomers and the chain-like protofibrils had disappeared and been replaced by two types of $A \beta$ fibrils: type-1 with a periodicity of $27.23 \pm 4.25 \mathrm{~nm}$ and a cross-sectional diameter of $4.60 \pm 0.56 \mathrm{~nm}$ and type-2 with a periodicity of $40.52 \pm 4.96 \mathrm{~nm}$ and a cross-sectional diameter of $4.07 \pm 0.46 \mathrm{~nm}$ (Fig. 1c). Protofibrils were occasionally observed on the surfaces of type-1 and type-2 fibrils (circles in Fig. 1c), pointing to the possible existence of secondary-nucleation sites. Type-2 fibrils were more abundant than type- 1 fibrils and exhibited a much higher frequency of secondary-nucleation sites. To assess the frequency and specificity of secondary nucleation, we quantified the number of secondary nucleation and type-2 fibrils in multiple images of the sample shown in Fig. 1 and Fig. S1a. Figure $1 f$ shows the different types of fibrils and the number of secondary-nucleation events observed for each fibril. For example, the total number of type-2 fibrils measured by AFM was 305 and the total number of secondary-nucleation events on type-2 fibrils was 105 , whereas only 5 secondary-nucleation events were observed in type- 1 fibrils $(n=35$; Fig. 1g). The number of secondary-nucleation events on the surface of type-2 fibrils decreased after $48 \mathrm{~h}$ of incubation and new types of fibrils (type-3 and wider fibrils) appeared after $48 \mathrm{~h}$ incubation, consistent with secondary nucleation being a secondary event. It could be possible that different types and sizes of fibrils have different affinities to the substrate. These are not exact quantitative observations of secondarynucleation events. However, as seen in Fig. 1f, it provides an insight of the progression toward new types of fibrils. Figure S1a illustrates the high occurrence of type-2 specific secondary nucleation under these conditions. After an incubation period of $68 \mathrm{~h}$, the protofibrils sprouting from the surfaces of 

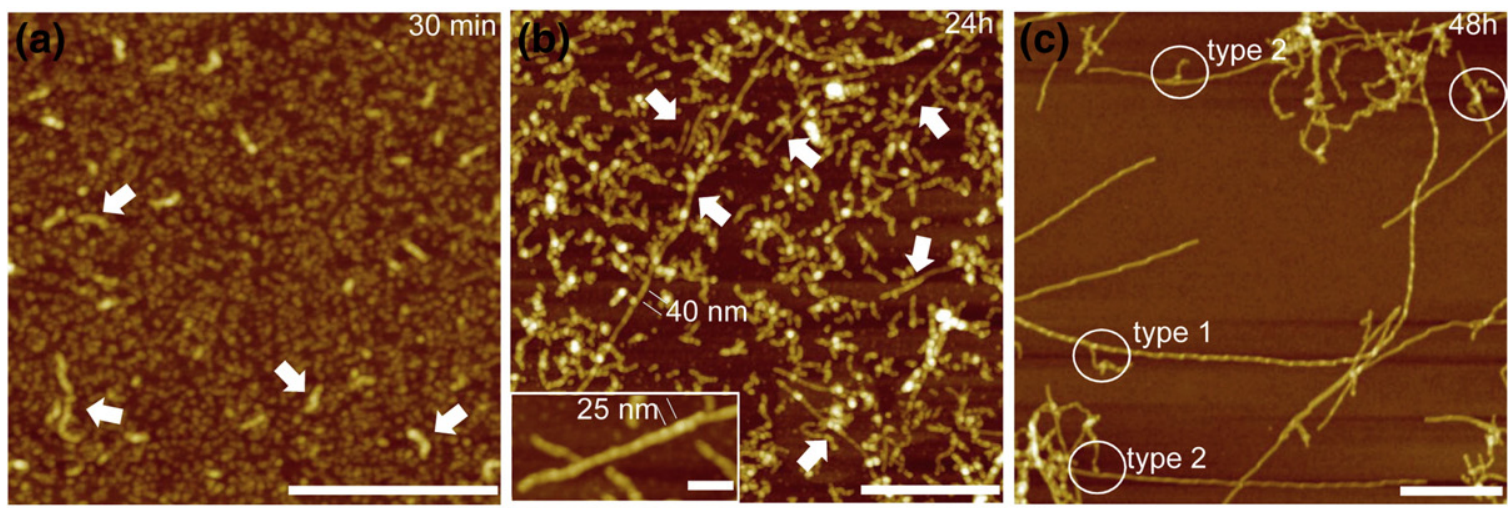

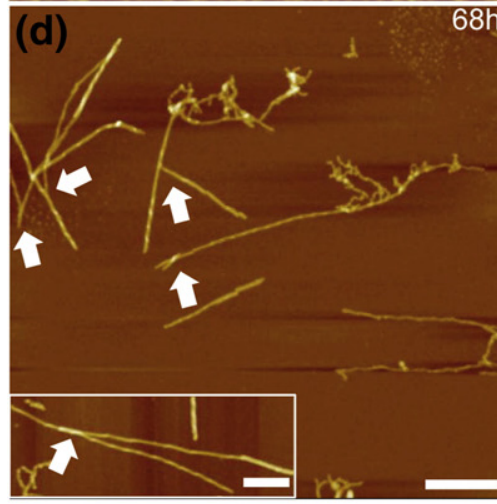

(g)

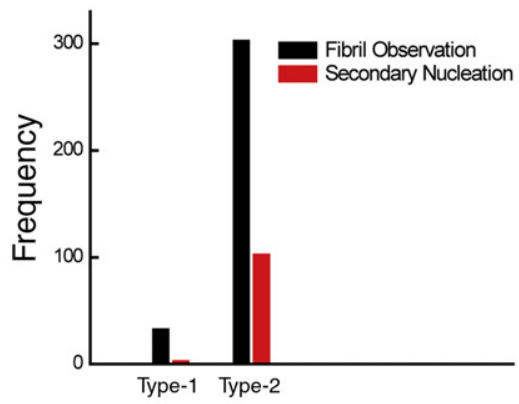

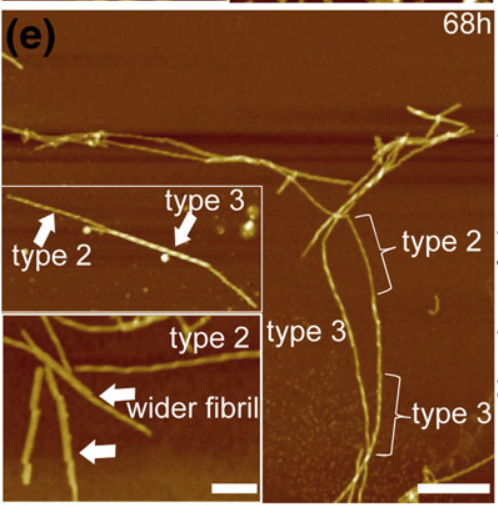

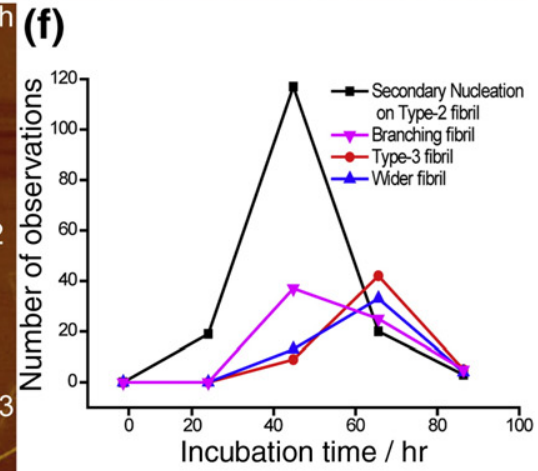

(h)

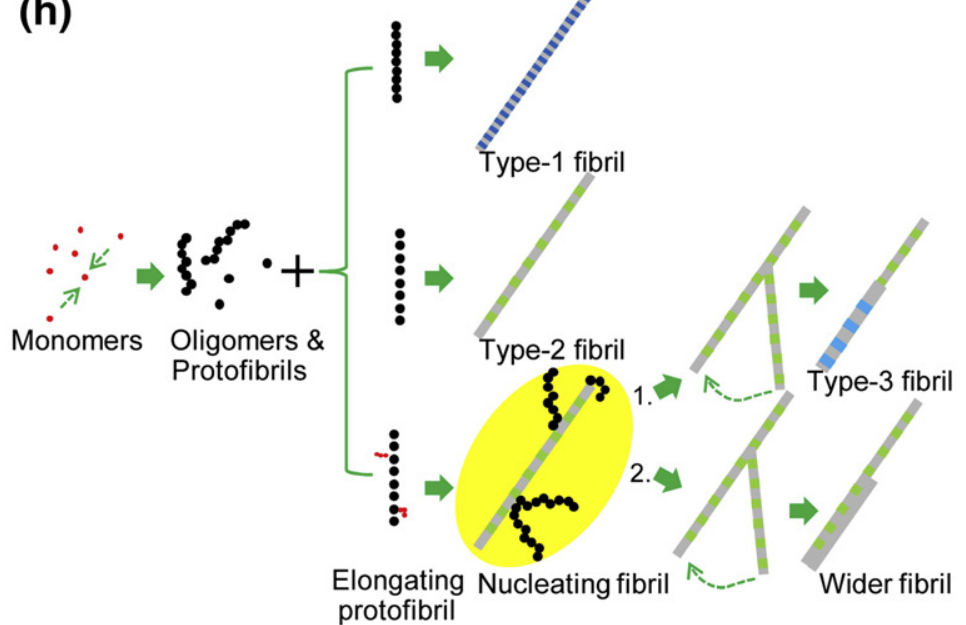

Fig. 1. Amyloid formation from monomeric Aß42 (Aß42-M). Incubation periods of (a) 30 min, (b) 24 h, (c) 48 h, (d) 68 h, and (e) 68 h. (f) The number of observations counts on secondary nucleation on type-2, branching, type-3, and wider fibrils at 24 h, 48 h, 72 h, and 96 h of incubation time. (g) Secondary-nucleation site counts on type-1 and type-2 fibrils. (h) Model of fibrillogenesis from Aß42-M. Scale bars represent $250 \mathrm{~nm}$. The $Z$ scale represents $25 \mathrm{~nm}$.

type-2 fibrils had evolved into fibrils, which exhibited the morphological characteristics and dimensions of the parent structure (Fig. 1d and Fig. S1b). In addition, a third type of fibril was observed. The periodicity of this type-3 fibril $(42.78 \pm 0.76 \mathrm{~nm})$ was similar to that of the type-2 fibril, but its crosssectional diameter $(7.54 \pm 1.00 \mathrm{~nm})$ was twice as great (Fig. 1e). However, wider fibrils (cross-sectional diameter: $4-5 \mathrm{~nm}$ ), with a smooth surface contour
(Fig. 1e), were frequently observed in conjunction mainly with type-2 fibrils. Figures S2a and S3 provide a quantitative summary of the height and periodicity property of each species measured by AFM during amyloid formation from A $342-\mathrm{M}$.

On the basis of repeated measurements, we propose the following model for fibril formation from Aß42-M (Fig. 1h). Spherical oligomers appear to be the primary building blocks for the aggregation of 
Aß42-M. Within $24 \mathrm{~h}$, these give rise to short-beaded chains of protofibrils via a process of linear elongation. The protofibrils then undergo self-assemblage to form type- 1 and type-2 fibrils. The type- 1 fibrils were characterized by the same cross-sectional diameter $(\sim 4 \mathrm{~nm})$ and periodicity $(\sim 25 \mathrm{~nm})$ as the protofibrils. This finding suggests that type-1 fibrils are formed exclusively by a process of which involves the sequential addition of monomers to form straight, rod-like structures without any change in cross-sectional diameter. Interestingly, the predominating type-2 fibrils have the same crosssectional diameter as the type-1 fibrils but a different periodicity $\sim 40 \mathrm{~nm}$. The detection of each fibril type at early timepoints-when protofibrils of similar dimensions dominate (Fig. 1b) - suggests that type-1 and type-2 fibrils reflect two distinct mechanisms of protofibril association. Type-2 fibrils can evolve into type- 3 and wider fibrils via a combined mechanism of secondary nucleation and lateral association. As shown in Fig. 1g, the AFM observations suggest that the surface of type-2 fibrils is the most probable secondary-nucleation sites. It is conceivable that AB42-M favors binding to type-2 fibrils. However, we note here that we cannot rule out an assembly pathway where spherical oligomers form independently and then stick to preformed fibrils either in solution during incubation or in AFM sample preparation. The disappearance of branching type-2 fibrils and the emergence of type-3 fibrils suggest that the latter are formed by the stacking of two type-2 fibrils. However, wider fibrils appeared to sprout from the surfaces of type-2 fibrils.

\section{Secondary-nucleation events confirmed by the kinetic analysis with ThT assay}

A sigmoid function as a function of time with thioflavin T (ThT) signal always implies the generation of new aggregates during the aggregation by the secondary pathways (fibril fragmentation or surfacecatalyzed nucleation). ${ }^{24,25}$ As reported by other studies, ${ }^{24-32}$ a reaction dominated by secondary nucleation undergoes an exponential increase in the early stages, as a result of the positive feedback. The positive feedback is a consequence of the fact that formed aggregates accelerate the rate of production of further aggregates. ${ }^{24}$

In order to support our morphological evidence of secondary-nucleation events on the surface of preexisting fibrils, we monitored the ThT fluorescence assay of AB42-M over time at three different protein concentrations. Figure $2 \mathrm{a}$ shows the aggregation reaction monitored by ThT fluorescence data of $A \beta 42-M$ at the different concentrations, exhibiting sigmoid form as a function of time with a distinct lag phase at low concentrations ( $10 \mu \mathrm{M}$ and $20 \mu \mathrm{M})$. At high concentration $(40 \mu \mathrm{M})$, the lag phase was shortened with increasing protein concentration.
The continuous lines in Fig. 2a was fitted according to

$$
S=\frac{S_{\infty}}{1+\mathrm{e}^{-\left(\left(t-t_{50}\right) / \tau\right)}}
$$

where $S_{\infty}$ is the amplitude of signal change, $t$ is the time, $t_{50}$ is the time at which the change in signal is at $50 \%$, and $\tau$ is a time constant. ${ }^{25}$ The lag time $\left(t_{D}\right)$ was calculated using the formula $t_{D}=t_{50}-2 \tau$ as described in Nielsen et al. ${ }^{30}$ Note that Eq. (1) is an empirical sigmoid fit function, and thus, the constants do not have an interpretation in terms of rates of molecular processes. Our data with increasing initial protein concentration (Fig. 2a) were well fitted by the sigmoid growth corresponding to Eq. (1). For the clear justification of secondary pathway, the initial $50 \%$ of the signal intensity with concentrations $10 \mu \mathrm{M}$ and $20 \mu \mathrm{M}$ was fitted to an exponential time function (continuous line in Fig. $2 \mathrm{~b}$ and $\mathrm{c}$ ) and a quadratic function (broken line in Fig. $2 b$ and c). It was reported by others ${ }^{24,25}$ that the primary nucleation pathway is following the quadratic in time, $\sim t^{2}$, while the reaction dominated by secondary nucleation pathway undergoes an exponential increase in the early stages of the reaction. ${ }^{24}$ The initial parts of our data with a $20 \mu \mathrm{M}$ concentration, which was used for $A \beta 42$ fibrillogenesis study, were fitted well by an exponential time function but poorly fitted by the $t^{2}$ function. This implies that the $A \beta 42$ aggregation is not dominated by the primary pathway and that our kinetic data provide evidences of secondary nucleation pathways. In contrast, the data at $10 \mu \mathrm{M}$ concentration were well fitted to an exponential time function but did not show a significant difference with the $t^{2}$ function.

In addition, Fig. $2 \mathrm{~d}$ shows the plot of experimental estimated $t_{50}$ (half-time) values of the ThT fluorescence-monitored kinetics against protein concentration. The straight line was fitted with a linear relationship when plotted as a log-log plot using Eq. (2).

$$
\log t_{f} \propto-\frac{1+n}{2} \log c_{0}
$$

where $t_{f}$ is the time at which a given fractional extent $(f)$ of aggregation is reached and $c_{0}$ is the total protein concentration. ${ }^{24}$ Since Fig. $2 a-c$ shows the reaction dominated by secondary pathway, we could use the slope, $-\frac{1+n}{2}$, from Fig. $2 d$ using Eq. (2) in order to determine the reaction order. ${ }^{24}$ The slope in Fig. $2 \mathrm{~d}$ was -1.424 yielding an $n$ value, reaction order of the nucleation process, of $1.848 .{ }^{24}$ If $n=0$, it corresponds to a monomer-independent secondary pathway (filament fragmentation), whereas if $n>0$, it corresponds to a monomer-dependent process that implies a surface-catalyzed nucleation, creating the new aggregates at a rate that depends on the monomeric concentration and the existing aggregates. ${ }^{24}$ The positive value of $n$ from our result 

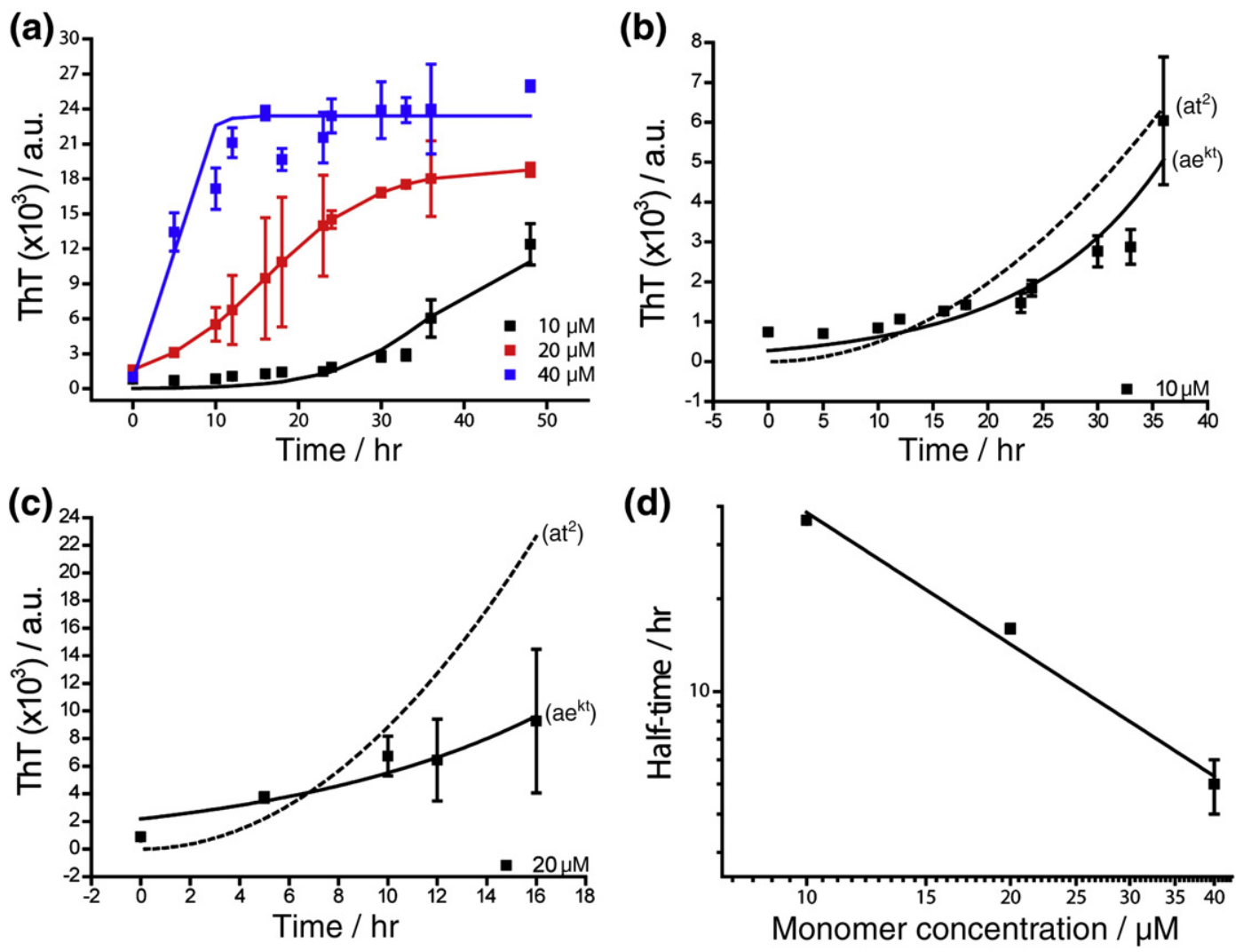

Fig. 2. Kinetics of amyloid fibril formation of an $A \beta 42-M$ sample at the concentrations of $10 \mu M, 20 \mu M$, and $40 \mu M$. (a) The ThT fluorescence data for increasing initial monomer concentration. The continuous line through the data points were fitted to Eq. (1). The initial $50 \%$ of the ThT fluorescence data of aggregation (b) $10 \mu \mathrm{M}$ and (c) $20 \mu \mathrm{M}$ were fitted to an exponential time function (continuous line) and a quadratic time function (broken line). (d) A plot of half-time values of ThT fluorescence against the initial monomer concentration. The continuous line through data points were fitted to Eq. (2). Slope: $-1.424 \pm 0.15$.

strongly supports that the secondary-nucleation events occurred on the surface of preexisting fibrils not just by fibril-fibril interaction.

\section{Amyloid formation from $A \beta 42$ protofibrils (Aß42-PF)}

In the absence of excess $A \beta 42-M$, purified $A \beta 42$ protofibrils (A $342-\mathrm{PF})$ do not aggregate into fibrils; they exist predominantly as curvilinear species (Fig. 3a), which grow with time but do not transform into mature ThT-positive amyloid fibrils (Fig. S4) ${ }^{33,34}$ Upon the addition of Aß42-M, the protofibrils undergo a rapid transition into amyloid fibrils. Hence, to investigate the transition from protofibrils to fibrils, we reintroduced $A \beta 42-M$ into solutions containing purified A $342-P F$ and examined the structural transitions and morphologies of the resulting fibrillar structures (Fig. $3 b-e$ ). When $A \beta 42-$ $M$ and $A \beta 42-P F$ were mixed, the ThT-binding value increased with time (Fig. S4). The morphology of the fibrillar structures depended upon the M/PF ratio. At higher M/PF ratios (5/1), the fibrils observed after
$87 \mathrm{~h}$ resembled those formed during the fibrillogenesis from Aß42-M (Fig. S5), whereas at lower M/PF ratios (0.3/1), long protofilaments (arrows in Fig. $3 b$ ) and numerous protofibrils predominated (Fig. 3b). The individual protofilaments appeared to have a periodic "twist" at intervals of $\sim 100 \mathrm{~nm}$. Whether this "twist" reflects the arrangement of the $\beta$-sheets in the protofilaments is unclear. The average crosssectional diameter of the protofilaments $(2.53 \pm$ $0.72 \mathrm{~nm}$ ) is consistent with previous observations. ${ }^{35}$ Mixed fibrillar structures consisting of protofilaments and type-4 fibrils defined below were observed in the same sample, thereby indicating the existence of a direct relationship between the two structures (oval in Fig. 3b), as reported by Adamcik et al. ${ }^{36}$ After an incubation period of $72 \mathrm{~h}$, most of protofibrils had disappeared and been replaced by protofilaments (Fig. 3c). However, at this 72-h juncture, the average cross-sectional diameter of the protofilaments $(3.79 \pm 0.70 \mathrm{~nm})$ exceeded that observed at $48 \mathrm{~h}$ (arrows in Fig. 3c). After $216 \mathrm{~h}$, most of protofilaments had disappeared and been replaced by type- 4 fibrils, which exhibited a regular periodicity of $74.66 \pm$ 

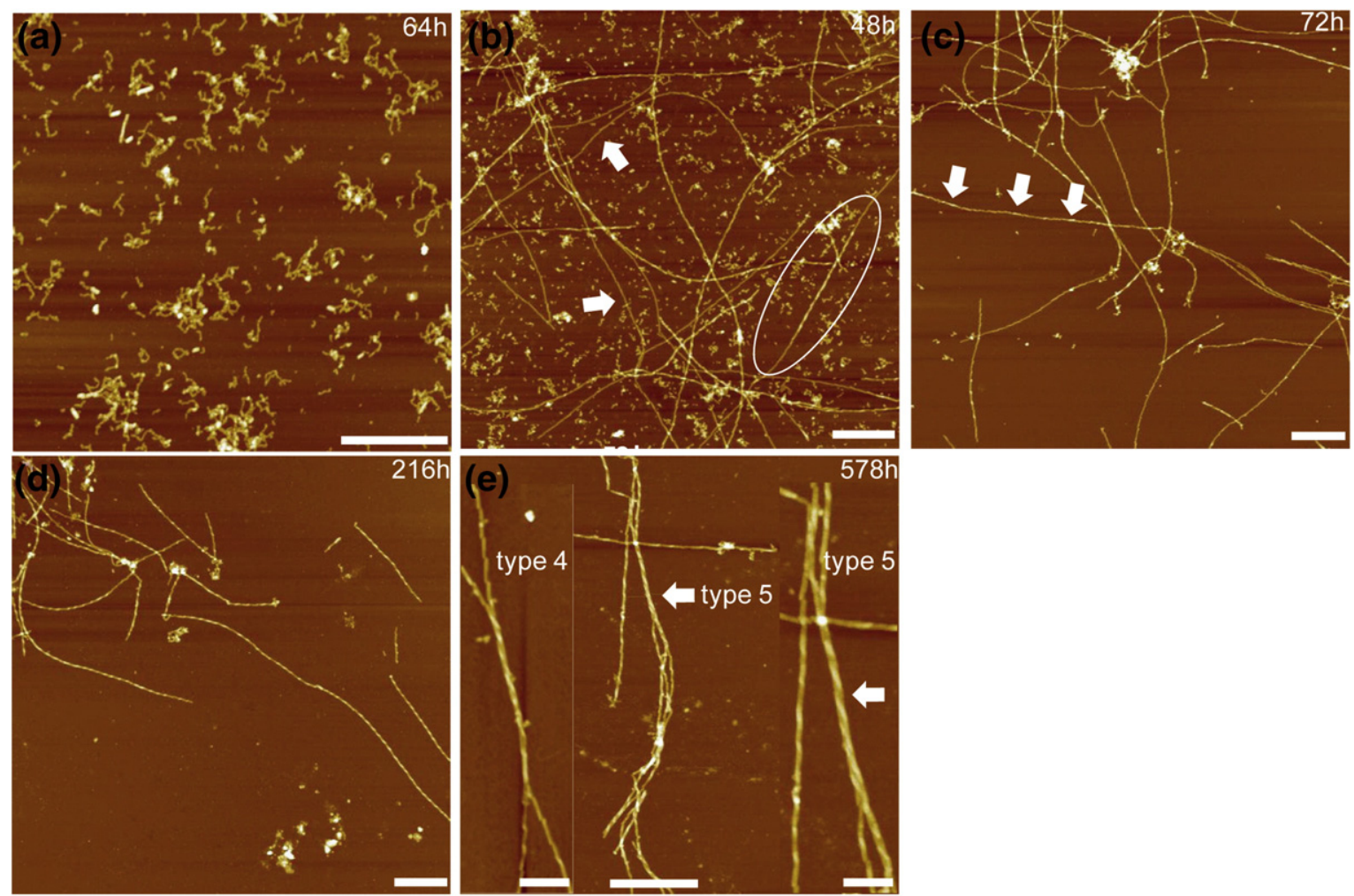

(f)
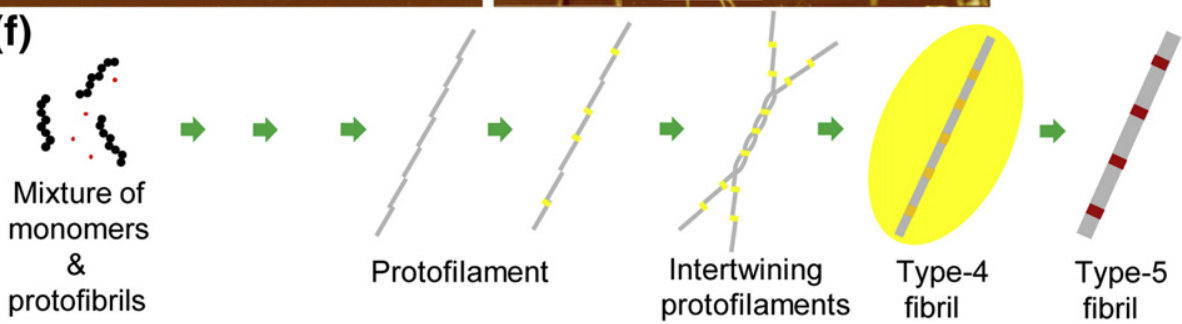

Fig. 3. Amyloid formation from $A \beta 42$ protofibrils (AB42-PF). (a) Purified $A \beta 42-P F$ after $64 \mathrm{~h}$ of incubation. A mixture of

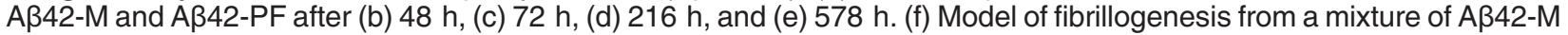
and Aß42-PF. Scale bars represent $500 \mathrm{~nm}(\mathrm{a}-\mathrm{e})$ and $100 \mathrm{~nm}$ (inset). The $Z$ scale represents $25 \mathrm{~nm}$.

$8.90 \mathrm{~nm}$ and a cross-sectional diameter of $7.29 \pm$ $0.82 \mathrm{~nm}$ (Fig. 3d). After an incubation period of $578 \mathrm{~h}$, we observed a small population of type- 5 fibrils, which were formed by the association of three protofilaments (Fig. 3e). However, at this juncture, and even after longer incubation times, type-4 fibrils predominated. Figure $3 e$ affords evidence by AFM of an intermediate stage of assemblage, involving the intertwining of two protofilaments to form a type-4 fibril and of three protofilaments to form a type-5 fibril (see Fig. S6). These structures (protofilaments and mixed fibrils) were observed only during amyloid formation from samples containing a mixture of $\mathrm{A} \beta 42-\mathrm{M}$ and $\mathrm{A} \beta 42-\mathrm{PF}$. We provided the table and histogram of height and periodicity property with the morphology of each species measured by AFM during amyloid formation from the mixture of $A B 42-M$ and PF. (Figs. S2b and S3).
On the basis of these observations, we propose a model of fibrillogenesis (Fig. 3f) in which the addition of monomers to protofibrils leads to the formation of protofilaments; the protofilaments self-associate and intertwine to form mature type-4 fibrils and, occasionally, after longer incubation times, type- 5 fibrils. Interestingly, these protofilaments were not observed during fibrillogenesis from AB42-M. Type-4 fibrils are highly twisted, and the twists occur at regular (periodic) intervals. Along the longitudinal axis of type-4 fibrils, a difference in cross-sectional diameter of $3.47 \pm 0.76 \mathrm{~nm}$ is observed between the maxima and the minima, ${ }^{36}$ which corresponds to the cross-sectional diameter of a protofilament $(3.79 \pm$ $0.70 \mathrm{~nm}$ ). Consistent with previous observations, ${ }^{35}$ the mature fibril is composed of two protofilaments. Taken together, these findings support our previous contention that the ratio of monomers to protofibrils is 
a crucial determinant of $A \beta$ aggregation and toxicity. ${ }^{33,34}$

\section{Other evidences for secondary-nucleation sites}

Our former studies on fibrillogenesis from $A \beta 42-M$ pointed to the existence of secondary-nucleation sites on the surfaces of amyloid fibrils. To confirm these findings and to ascertain whether secondary nucleation depends upon the structure and the morphology of the fibrils, we added $A B 42-M$ to samples containing either preformed type-2 fibrils or protofilaments, which were then incubated for a further $72 \mathrm{~h}$ or $47 \mathrm{~h}$, respectively.

The reintroduction of $A \beta 42-M$ into a solution containing preformed type-2 fibrils (Fig. 4) led to further growth at the secondary-nucleation sites and accelerated the process of fibrillogenesis; it also led to the emergence of type-3 (asterisk in Fig. 4) and wider (arrows in Fig. 4) fibrils, which arose by the lateral association of type-2 fibrils. No secondarynucleation events were observed on the surfaces of either the type-3 or the wider fibrils.

The reintroduction of $A \beta 42-M$ into a solution containing protofilaments led, after $24 \mathrm{~h}$, to the formation predominantly of thicker protofilaments (Fig. 5a), upon the surfaces of which no signs of secondary nucleation were detected on the surfaces of protofilaments. These findings support earlier observations indicating that only mature type-2 fibrils are capable of secondary nucleation fibrillogenesis on their surfaces.

After $23 \mathrm{~h}$, we observed a transition from protofilaments to mixed fibrillar structures, which included type-1, type-4, and type-5, with type-4 predominating (Fig. 5b). The type-4 and the type-5 fibrils observed under these conditions exhibited the same morphological features as those in samples containing a mixture of monomers and protofibrils (Fig. $3 d$ and e).

Based on these observations, we propose the following model of fibrillogenesis under conditions in which A $442-M$ and AB42-PF co-exist. Type-4 fibrils predominate under these conditions. Given the periodic difference in the cross-sectional diameter of type-4 fibrils $(3.50 \pm 0.54 \mathrm{~nm})$ and the morphological features of the transition stage (Fig. S6a), the former appear to be formed by the intertwining of two thicker protofilaments. Type-5 fibrils exhibit a periodicity of 90-150 nm along the longitudinal axis and have a cross-sectional diameter of $9.17 \pm 0.89 \mathrm{~nm}$. The latter measurement is consistent with their being composed of three thicker protofilaments. This conclusion is supported by the repeated observation of structures manifesting the merger of three thicker protofilaments to form a type- 5 fibril (Fig. S6b). The type- 1 fibrils revealed under these conditions exhibited the same morphological features as those observed during fibrillogenesis from $A \beta 42-M$ (Figs. 1c and 5c). Conceivably, the reintroduction of $A \beta 42-M$ led to a linear alignment of the peptides to form beaded chains with the periodicity of type-1 fibrils $(30.25 \pm 4.86 \mathrm{~nm})$. Accordingly, the population of type-1 fibrils increased after the reintroduction of $A \beta 42-M$ only during the course, not prior to the onset, of aggregation. Taken together, these findings demonstrate that the surfaces of fibrils can serve as templates for the replication of the parent structure.

\section{Real-time monitoring of the structural transition from oligomers to protofibrils}

Our findings indicate that $A \beta 42-M$ plays a crucial role in determining the pathway of $A \beta 42$ aggregation and the morphology of the final fibrils. To test this hypothesis, we investigated the structural transition from oligomers to protofibrils by in situ AFM. AB42 oligomers that had been generated from $A \beta 42-M$ after an incubation period of $4 \mathrm{~h}$ were first deposited upon 3-aminopropyl triethoxysilane (APTES)-modified mica (Figs. 6a, 7a, and 8a). These samples were rich in spherical structures, with an average diameter of 2.2-2.4 nm (Figs. 6a-1, 7a-1, and 8a-2) and in short assemblies with a similar cross-sectional
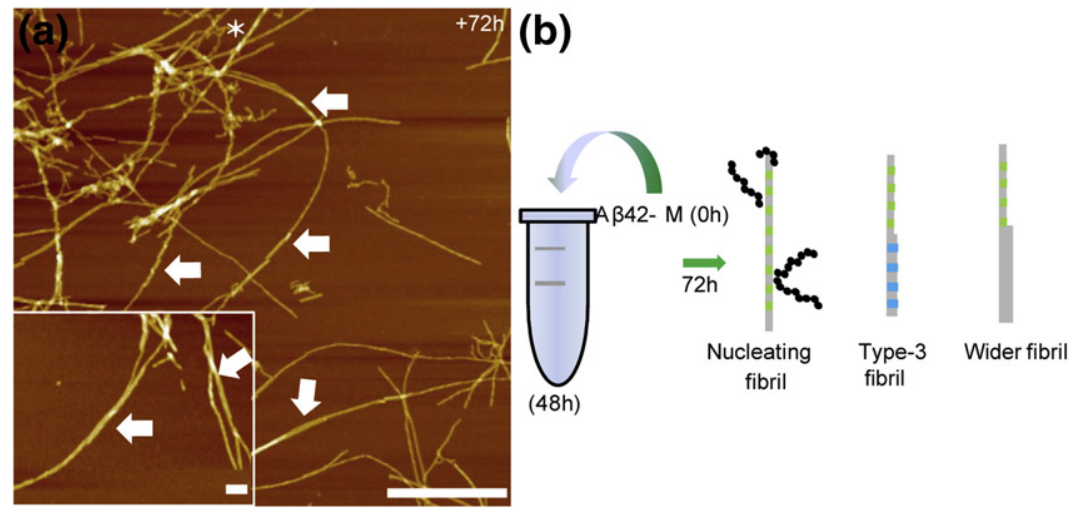

(48h)

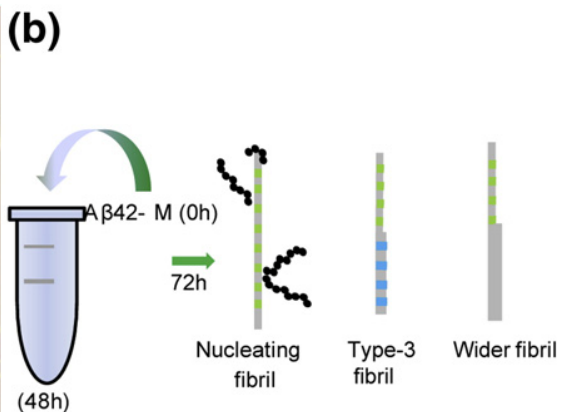
represent $500 \mathrm{~nm}$ and $90 \mathrm{~nm}$ (inset). The $Z$ scale represents $25 \mathrm{~nm}$. (b) Schematic representation of the experimental procedure and the results.
Fig. 4. (a) AFM image of amyloid formation from $A \beta 42-M, 72 \mathrm{~h}$ after reintroducing $A \beta 42-M$ into the solution containing preformed type-2 fibrils (48-h-incubation sample). This measure led to further growth and fibrillogenesis at the secondary-nucleation sites, to an acceleration of the latter process, and to the emergence of type-3 (asterisk) and wider (arrows) fibrils. Scale bars 

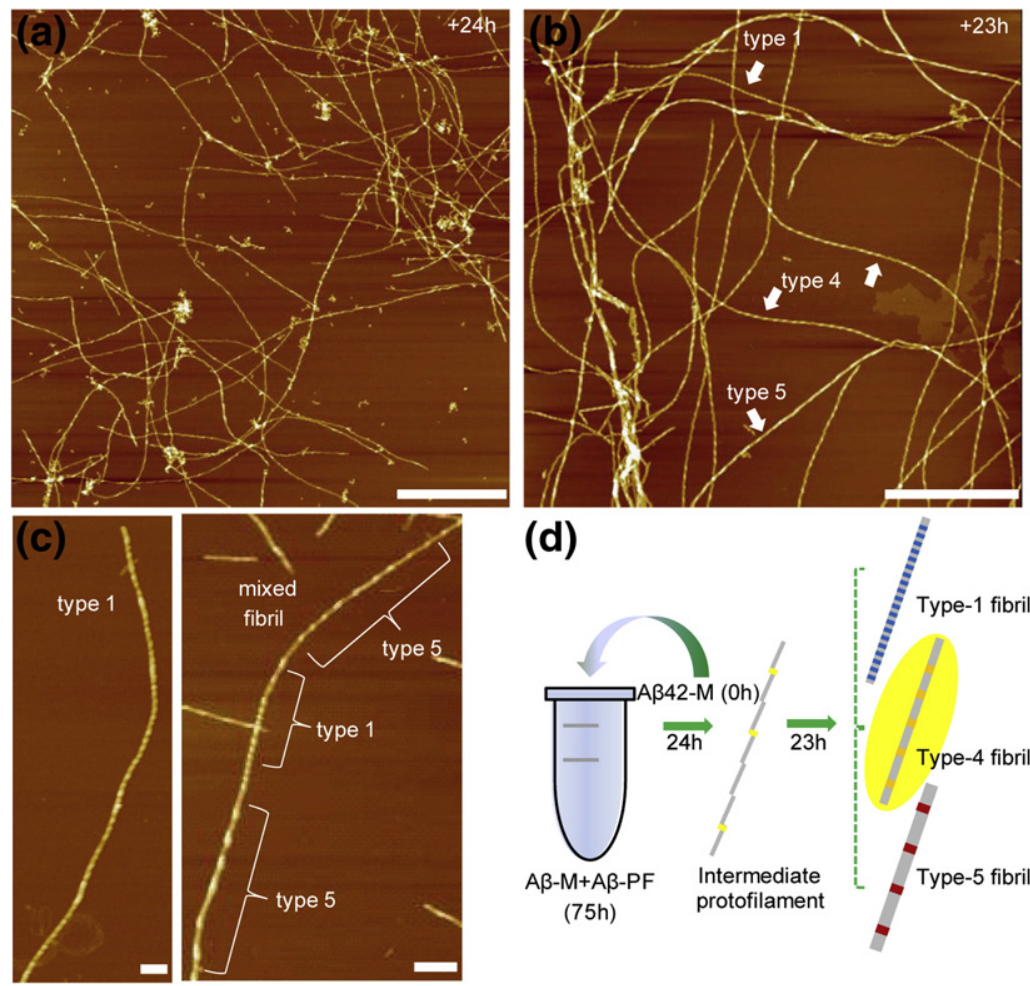

(d)

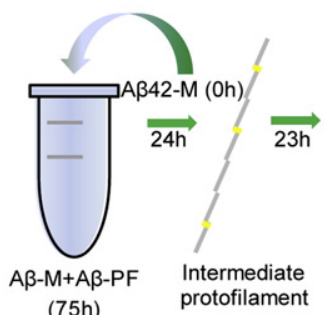

(75h)

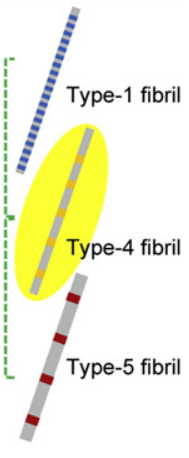

Fig. 5. (a) AFM image of amyloid formation from a mixture of $A \beta 42-M$ and $A \beta 42-P F, 24 \mathrm{~h}$ after reintroducing $A \beta 42-M$ into the solution containing mainly protofilaments (75-hincubation sample). At this juncture, predominantly thicker protofilaments were observed. (b) After an additional $23 \mathrm{~h}$, type-1, type-4, and type- 5 fibrils were detected, with the type-4 variety predominating. (c) The morphological features of the type-1 fibrils that were ascertained (left). Fibrils combining the properties of type-1 and type-5 were observed (right), but no evidence of secondary nucleation was detected. Scale bars represent $500 \mathrm{~nm}$ (a and b) and $90 \mathrm{~nm}$ (c). The $Z$ scale represents $25 \mathrm{~nm}$. (d) Schematic representation of the experimental procedure and the results. diameter and a length of $\sim 20 \mathrm{~nm}$. We then examined the dynamic (real-time) growth of specific oligomers after the injection of either freshly prepared $A \beta 42-\mathrm{M}$ (Fig. 6), oligomers (Fig. 7), or Aß42-PF (Fig. 8) into the fluid cell. Our data revealed the rate of oligomer growth to be most rapid after the introduction of A $342-\mathrm{M}(9.9 \pm 2.64 \mathrm{~nm} / \mathrm{min})$, somewhat slower after the injection of $A \beta 42-P F(1.44 \pm 0.34 \mathrm{~nm} / \mathrm{min})$, and slowest after the addition of oligomeric $A \beta 42(0.75 \pm$ $0.25 \mathrm{~nm} / \mathrm{min}$ ). Forty minutes after the injection of $\mathrm{A} \beta 42-\mathrm{M}$, the oligomers underwent a smooth transformation into elongated structures with a crosssectional diameter ( $2 \mathrm{~nm}$; Fig. $6 \mathrm{~b}-1)$ similar to that of the oligomers and a length ranging from 150 to $400 \mathrm{~nm}$. No further growth was observed after $3 \mathrm{~h}$. An additional injection of $A B 42-M$ led to a further elongation of the structures and an increase in their cross-sectional diameter ( 4.5 nm; Fig. 6c-1).

When a 4-h-incubation solution containing a mixture of oligomers and A $A 42-\mathrm{M}$ was added (Fig. 7), we observed two main structural changes: (1) an increase in the cross-sectional diameter of the spherical oligomers (upper histogram in Fig. 7b-1) and (2) the emergence of elongated oligomers with a cross-sectional diameter of $\sim 2 \mathrm{~nm}$ (lower histogram in Fig. 7b-1) and a length ranging from 45 to $430 \mathrm{~nm}$. Predeposited oligomers rarely underwent elongation (asterisk in the insets) - a finding that is consistent with the low concentration of $A \beta 42-M$ in these preparations. Figure 7 reveals that the elongated oligomers originated from the predeposited spherical ones (circles in the insets).
We also attempted to ascertain whether preformed protofibrils were capable of associating and growing upon the surfaces of predeposited oligomers, but the protofibrils did not bind well to the positively charged surfaces (Fig. 8). However, the spherical oligomers in these preparations (Fig. 8a and a-1) were observed to associate and grow upon the surfaces of preexisting spherical-to-elongated oligomers (Fig. 8b and b-1). Figure $8 \mathrm{~b}$ reveals that, $4 \mathrm{~h}$ after the injection of a solution containing protofibrils, the spherical oligomers underwent elongation without a change in crosssectional diameter. The growth of the oligomers followed the orientation of the ordered trigonal units comprising the mica surface. ${ }^{37}$ The oligomers grew to form elongated oligomers with a length that did not exceed $300 \mathrm{~nm}$. Forty-five minutes after the injection of freshly purified AB42-M into the fluid cell, the crosssectional diameter of the observed elongated oligomers (Fig. 8b-1) increased to $4 \mathrm{~nm}$ (Fig. 8c-1 and c-2), which is close to that measured by AFM in air (Fig. 1b). Figure $8 \mathrm{c}-1$ reveals the beaded, chain-like structure of the protofibrils. The consecutive series of images in Fig. 9 depict in real time the structural transition from elongated oligomers to protofibrils and the corresponding changes in cross-sectional diameter. At early incubation times, the newly deposited monomers formed beaded structures upon the preexisting assemblies. These beaded structures gradually transformed into smooth-contoured structures with a cross-sectional diameter that was twice as great as that of the oligomers (see also Fig. S7). These observations support the hypothesis that $A \beta 42$ 

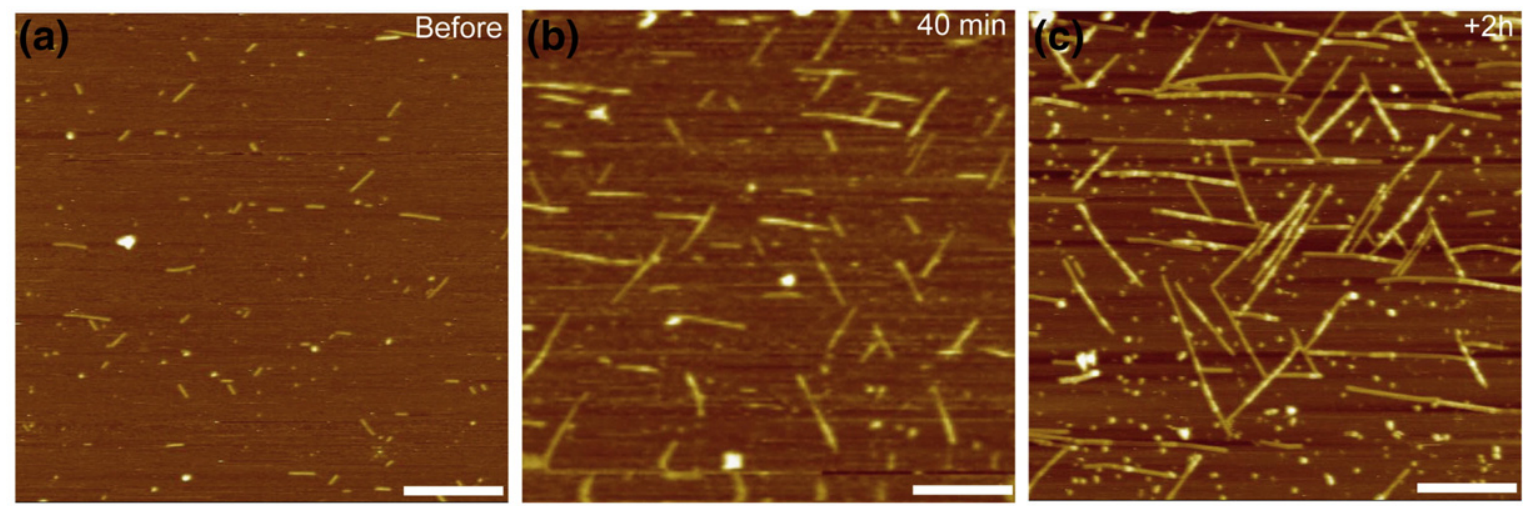

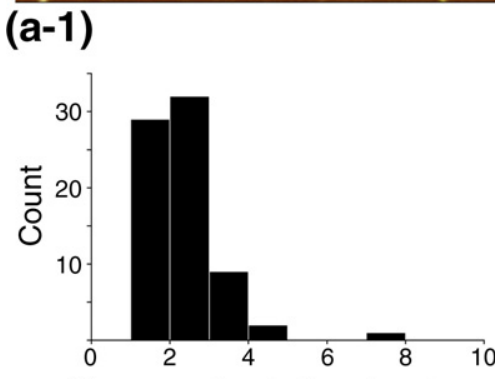

Cross-sectional diameter of oligomers \& short assemblies $(\mathrm{nm})$ $(\mathrm{b}-1)$

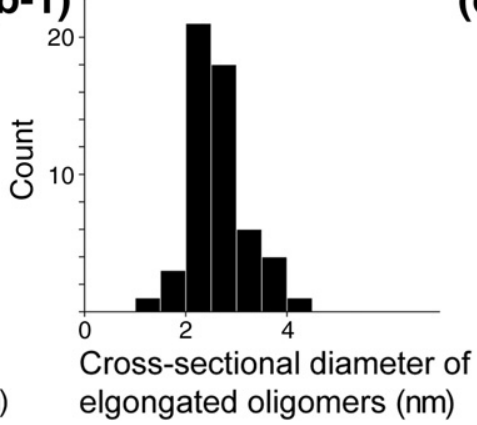

$(\mathrm{c}-1)$

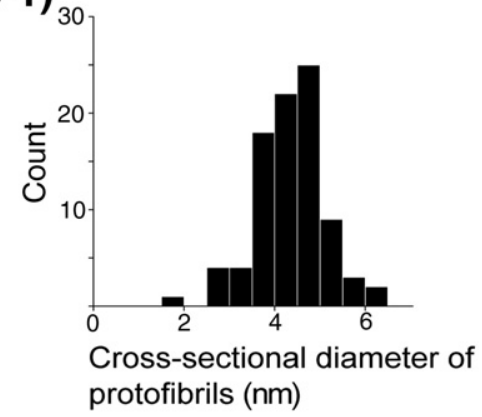

Fig. 6. Real-time AFM images illustrating the structural transition from oligomers to protofibrils, together with histograms depicting changes in the numerical distribution of the cross-sectional diameters of the linear structures (a-1, b-1, and c-1). (a) Before the addition of fresh A $42-\mathrm{M}$, spherical oligomers and short linear assemblies of these, with a smooth surface contour and a cross-sectional diameter of $\sim 2.0 \mathrm{~nm}$, were observed. (b) Forty minutes after the addition of fresh Aß42-M to the mica substrate, the short linear assemblies of oligomers had undergone elongation at a rate of $9.9 \pm 2.64 \mathrm{~nm} / \mathrm{min}$; $\mathrm{no}$ further elongation occurred after $3 \mathrm{~h}$. (c) Two hours after a second addition of Aß42-M, the linear structures had undergone further elongation, and their cross-sectional diameter had increased from $\sim 2.0 \mathrm{~nm}$ to $\sim 4.5 \mathrm{~nm}$. Scale bars represent $250 \mathrm{~nm}$. The $Z$ scale represents $10 \mathrm{~nm}$.

monomers play an important role in mediating the association of oligomers and the growth of protofibrils (Fig. 9b). However, the branching points where two elongated structures encountered (Figs. 6-8) were not generated by secondary-nucleation events as shown in Fig. 1. It needs to be stressed here that, owing to the constraints imposed by the substrate, ${ }^{17}$ neither the lateral association of protofibrils nor the formation of twisted fibrils was observed in the in situ experiments.

\section{Discussion}

To understand the role played by $A \beta$ aggregation in the pathogenesis of Alzheimer's disease, and thus to be in a position to develop effective strategies for its treatment, it is necessary to have a clear conception of the molecular mechanisms underlying amyloid formation and the contribution of each intermediate. Although previous studies have shown that $A \beta$ peptides can self-assemble into polymorphic fibrillar structures, the mechanisms underlying this process and the relationship existing between the different fibrillar forms of $A \beta$ are poorly understood. In vitro, the morphology of the final fibrils and the extent of their heterogeneity (in terms of diameter, length, and the degree of twisting) depend not only up on the amino acid sequence but also upon many other factors, including the absence or presence of agitation during the process of aggregation, ${ }^{38,39}$ protein concentration, ${ }^{14,20}$ the physical properties $\left(\mathrm{pH}^{15}\right.$ and temperature $\left.{ }^{16}\right)$ and composition of the incubation medium (nature of the buffer, ${ }^{15}$ salt concentration, ${ }^{15,17}$ and the absence or presence of a detergent ${ }^{15}$ ), the type of seeding species, ${ }^{13,18,38}$ and the mode of microscopic analysis. ${ }^{13,19}$ Single protein molecules of, for example, $A \beta,{ }^{40}$ amylin, ${ }^{41}$ insulin, ${ }^{42}$ or calcitonin, ${ }^{14}$ are indeed known to exhibit a broad diversity of morphologies under the same incubation conditions. Petkova et al. have suggested that the polymorphism of $A \beta 40$ fibrils might be attributable either to variations in the molecular structure of the protofilaments or to differences in their mode of lateral association that would have no impact on their molecular structure. ${ }^{38}$ Others have proposed that conformational differences at the level of the 

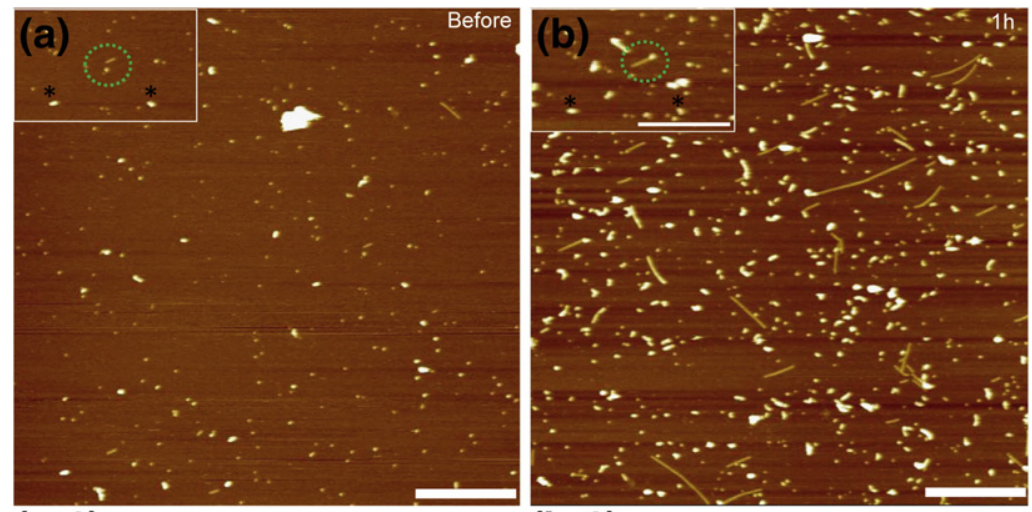

(a-1)

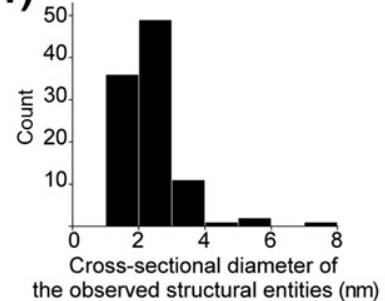

(b-1)

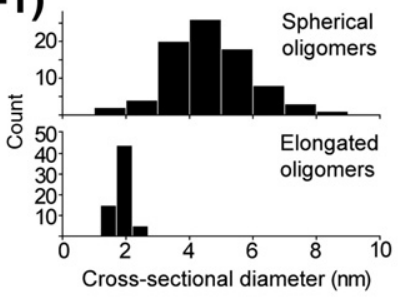

Fig. 7. Real-time AFM images illustrating the structural transition of oligomers, together with histograms depicting changes in the numerical distribution of crosssectional diameters of the observed structural entities (a-1 and b-1). (a) Before the addition of oligomeric A $\beta 42$, spherical oligomers (asterisk in inset) and short linear assemblies of these (circle in inset), with a smooth surface contour and a cross-sectional diameter of $\sim 2 \mathrm{~nm}$, were observed. (b) One hour after the addition of fresh oligomeric Aß42 (to a 4-h-incuation sample), the cross-sectional diameter of the spherical oligomers had increased (upper histogram), and the short linear structures had undergone elongation at a rate of $0.75 \pm 0.25 \mathrm{~nm} / \mathrm{min}$, without a change in cross-sectional diameter (circle in inset and lower histogram). Scale bars represent $500 \mathrm{~nm}$ and $100 \mathrm{~nm}$ [inset in (b)]. The $Z$ scale represents $10 \mathrm{~nm}$.

monomeric protein ${ }^{39}$ or in the manner in which the early oligomers assemble ${ }^{17}$ prior to protofilament formation could account for the polymorphic nature of $A \beta$ fibrils. The importance of improving our understanding of the molecular and structural basis of fibrillar heterogeneity has been recently brought home by indications that the various strains of disease-inducing prions arise as a result of differences in fibrillar structure. This finding accords with observations that fibrils with different morphologies exhibit distinct properties of resistance to proteases $^{43}$ and chemical denaturants ${ }^{44}$ and of seeding efficiency. ${ }^{45}$ In some cases, the fibrils also differ in molecular structure and in their dye-binding properties. ${ }^{46}$

In the present study, we investigated amyloid formation and the structural properties of $A \beta 42$ species. In particular, we addressed the influence of the following: (1) the aggregation state of the starting material (monomers, oligomers, or protofibrils), (2) the concentration of the monomeric protein at different stages of amyloid formation, (3) incubation time, and (4) secondary-nucleation events. Previous TEM studies have indicated that the type of negative staining can influence the morphology of $A \beta$ fibrils. ${ }^{13,14,19}$ In contrast to TEM, the visualization of structures by AFM does not require them to be stained. Hence, we used this methodological tool to explore the molecular topography of the structural entities that are generated during amyloid formation and to monitor the dynamics of this process in solution under conditions that approach those pertaining in vivo. ${ }^{17,21,47-49}$ Hitherto, most AFM studies relating to
$A \beta$ have involved the use of $A \beta 40 ; A \beta 42$ has been employed but rarely. ${ }^{48,50,51}$ We have taken advantage of the reproducible protocols that have been developed in our laboratory ${ }^{52}$ for the preparation of monomeric, protofibrillar, and fibrillar A 342 to address the aforementioned issues.

\section{Molecular basis of the polymorphism of $A \beta 42$ fibrils}

Although the precise mechanism governing $A \beta 42$ aggregation in vivo remains uncertain, the data presented in the present study have afforded a novel insight into the process and have led us to propose a mechanistic model for the formation, growth, and self-assemblage of $A \beta 42$ fibrils in vitro. Our findings demonstrate that the oligomeric state of the starting material and the relative concentrations of the different $A \beta 42$ species (monomers, oligomers, and protofibrils) have a profound influence on the polymorphism and the structural heterogeneity of the resulting fibrils. Using purified and homogeneous preparations of monomeric $A \beta 42$ (AB42-M), we observed this species to undergo self-assemblage initially into spherical oligomers and subsequently into beaded, chain-like protofibrils with different periodicities $(\sim 25 \mathrm{~nm}$ or $\sim 40 \mathrm{~nm})$ but the same cross-sectional diameter $(\sim 4 \mathrm{~nm})$. These protofibrils then gave rise mainly to type- 1 and type-2 fibrils. However, we also observed type-3 and wider fibrils, which are formed by the lateral association or stacking of two type-2 fibrils. 


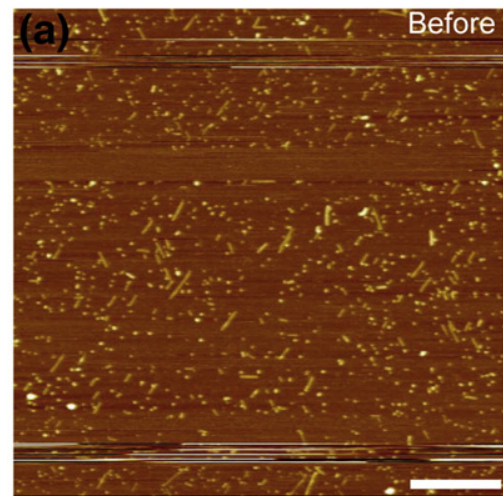

(a-1)
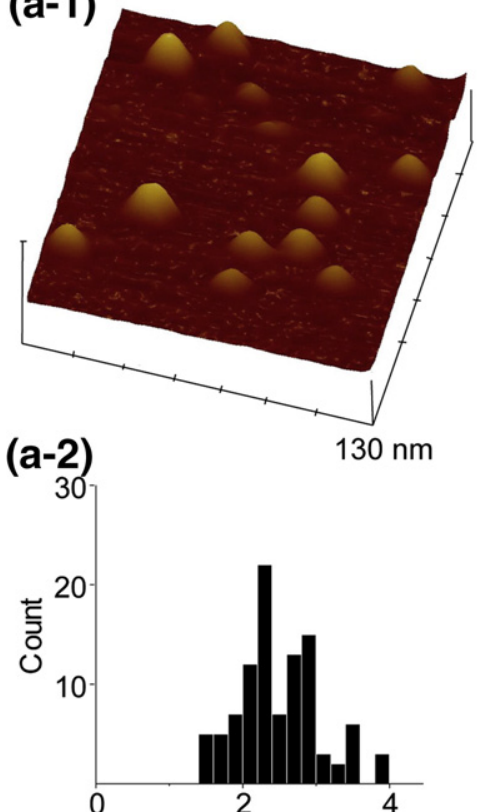

Cross-sectional diameter of oligomers \& short assemblies (nm)
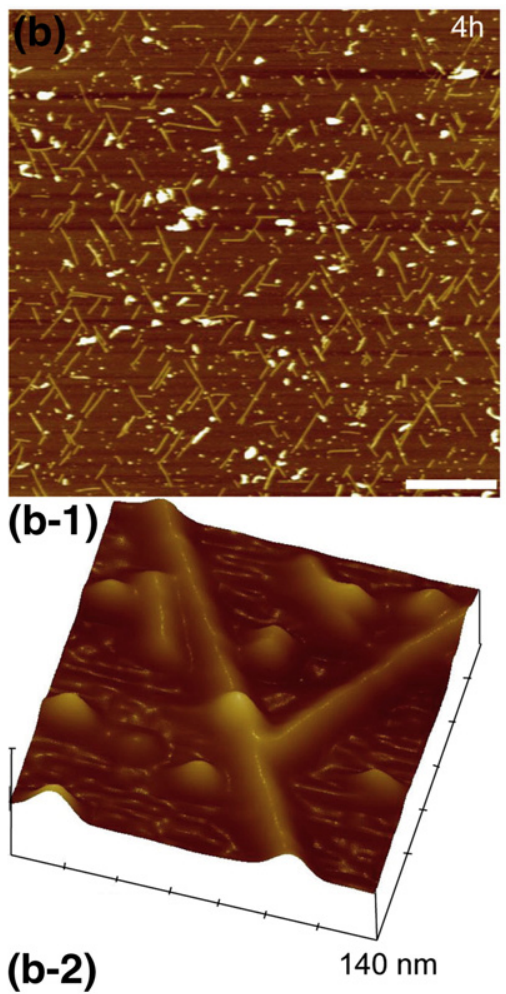

(b-2)

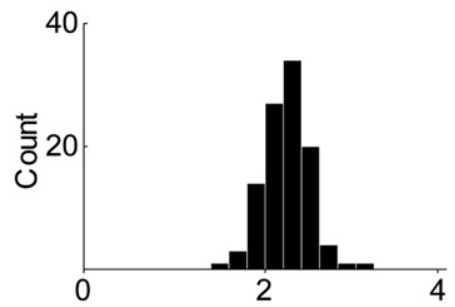

Cross-sectional diameter of elongated oligomers $(\mathrm{nm})$

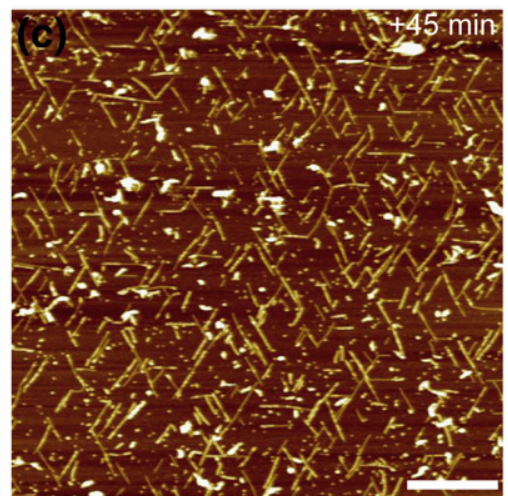

(c-1)
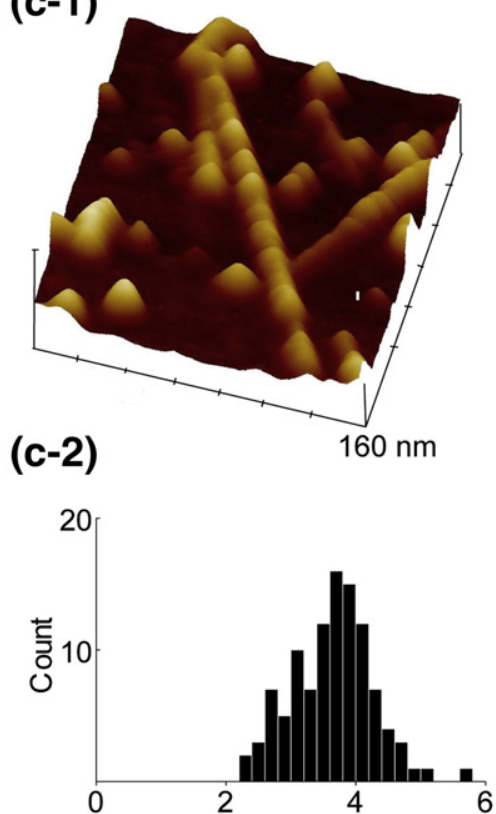

Cross-sectional diameter of protofibrils $(\mathrm{nm})$

Fig. 8. Real-time AFM images $(a-c)$ and the corresponding three-dimensional reconstructions (a-1, b-1, and c-1) of the structural transition from oligomers to protofibrils, together with histograms depicting changes in the numerical distribution of cross-sectional diameters of the linear structures (a-2, b-2, and c-2). (a, a-1, and a-2) Before the addition of Aß42-PF, spherical oligomers and short linear assemblies of these, with a smooth surface contour and a cross-sectional diameter of $\sim 2 \mathrm{~nm}$, were observed. (b, b-1, and b-2) Four hours after the addition of Aß42-PF (24-h-incubation sample), the short linear structures had undergone elongation at a rate of $1.44 \pm 0.34 \mathrm{~nm} / \mathrm{min}$, without a change in cross-sectional diameter. (c, $\mathrm{c}-1$, and c-2) Forty-five minutes after the addition of AB42-M, the linear structures had undergone further elongation, and their cross-sectional diameter had increased from $\sim 2.0 \mathrm{~nm}$ to $4.0 \mathrm{~nm}$. (c-1) illustrates particularly well the bead-like nature of a linear assemblage of spherical oligomers. Scale bars represent $500 \mathrm{~nm}$. The $Z$ scale $(\mathrm{a}-\mathrm{c})$ represents $10 \mathrm{~nm}$, and that in (a-1), (b-1), and (c-1) represents $6.5 \mathrm{~nm}$.

In previous studies, we have demonstrated that, in the absence of measurable amounts of monomeric $A \beta 42$, purified protofibrils elongate very slowly and fail to form mature fibrils. ${ }^{33,34}$ Upon the addition of excess monomeric $A \beta 42$, protofibrils undergo rapid conversion to fibrils. Hence, we assessed the formation of fibrils from protofibrils in the presence of different concentrations of AB42-M. When fibrillogenesis was initiated using a mixture of $A \beta 42-M$ and $A \beta 42-P F$, predominantly mature, type-4 fibrils were formed. Both the degree and the rate of conversion of protofibrils to fibrils rose with an increase in the concentration of $A \beta 42-M$. Under these conditions, we observed the intermediate subfibrillar structures that associate to form the protofilaments then two or three of thicker protofilaments intertwine to form stable type-4 or type-5 fibrils, respectively. No secondary-nucleation events were observed upon the surfaces of either type- 4 or type- 5 fibrils. Furthermore, when fresh monomeric $A \beta 42$ was 
(a)
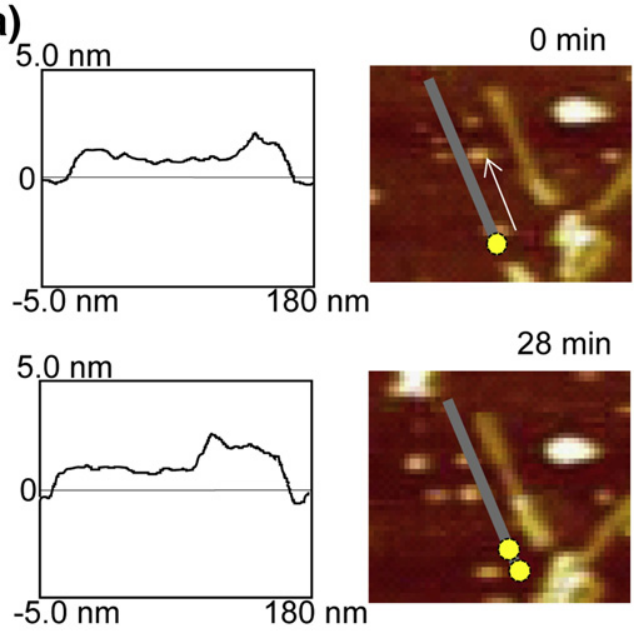

$38 \min$
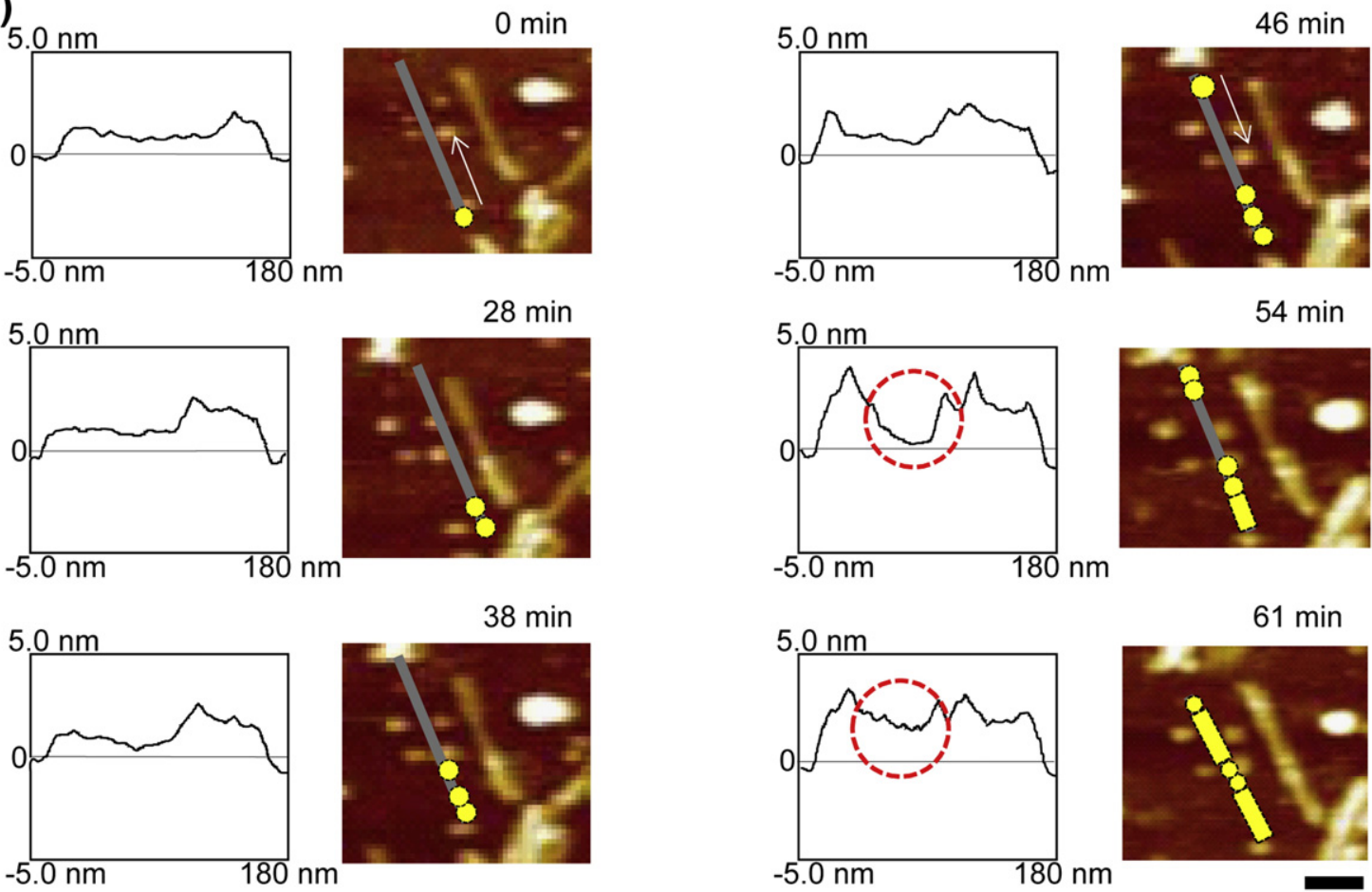

$54 \min$

(b)

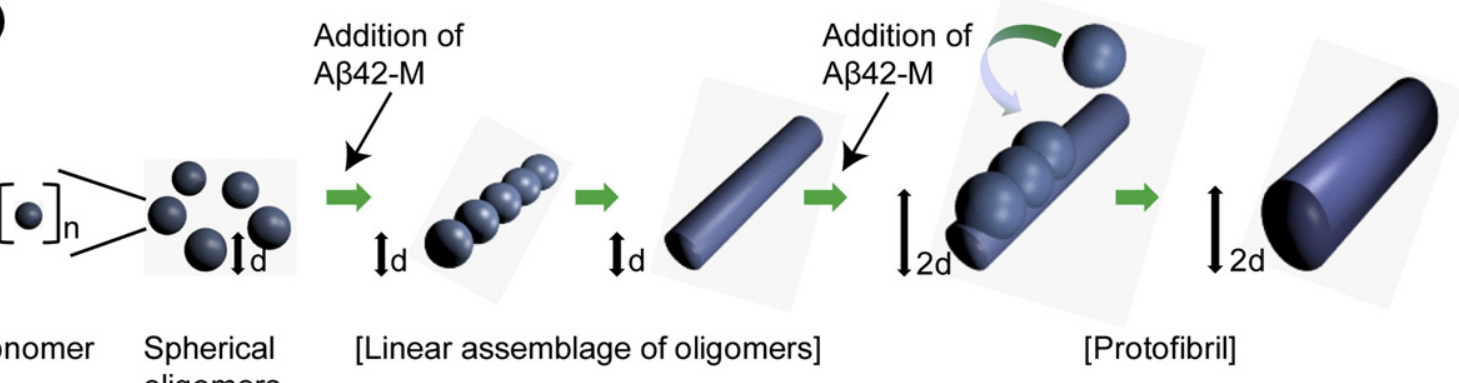

oligomers

*d: cross-sectional diameter

Fig. 9. (a) Real-time AFM images (right) of the structural transition from an elongated oligomer to a protofibril, together with graphs tracing the change in cross-sectional diameter of the imaged structure. Twenty-eight minutes after the addition of $A \beta 42-M$ to the elongated oligomer that is imaged at timepoint zero (0 min), monomers had begun to attach to one end of the linear structure (yellow circles in the scheme of the imaged entity). After 38 min, the sequential addition of new monomers to the preexisting (aligned) ones had continued. After $46 \mathrm{~min}$, the process of monomer addition had begun also at the other end of the linear oligomer (white arrow). After $54 \mathrm{~min}$, the process of monomer addition had progressed. Between $54 \mathrm{~min}$ and $61 \mathrm{~min}$, the linear structures bearing the aligned monomer began to undergo a marked increase in cross-sectional diameter (see the circled regions in the graphs), which denoted their transformation into protofibrils. The scale bar represents $45 \mathrm{~nm}$. The $Z$ scale represents $5 \mathrm{~nm}$. (b) Model representing the transition from oligomers to protofibrils during in situ $\mathrm{A} \beta 42$ fibrillogenesis.

added to solutions containing predominantly protofilaments, type-1 fibrils were formed. This finding suggests that newly added monomers assemble independently and do not become incorporated into the protofilaments. Our data indicate that the polymorphism of $A \beta 42$ fibrils may arise (1) by their lateral association (yielding type-3 and wider fibrils) and (2) by the association of different numbers of protofilaments (yielding fibrils with different crosssectional diameters and periodicities, e.g., type-4 and type-5). Previous studies have shown that $A \beta 40$ forms polymorphic fibrillar structures and suggested that $A \beta 40$ polymorphism may be due to the existence of multiple assembly pathways ${ }^{17}$ that are strongly dependent on solution conditions (e.g., protein concentration, $\mathrm{pH}$, and growth condition). ${ }^{39}$ Oligomeric structures of similar dimensions to those reported here have been observed for $A \beta 40 .^{53}$ However, direct comparison of the kinetics and structural transitions between these different studies 
is not possible due to the large differences in the starting aggregation states of the protein and experimental conditions.

\section{Secondary nucleation is fibril-type specific}

Recent studies have indicated that, in addition to primary nucleation and elongation, the growth and assemblage of fibrils can occur via secondary nucleation upon the surfaces of specific, preexisting fibrils. ${ }^{54-56}$ Knowles et al. have proposed a model of biomolecular self-assemblage for insulin, $\beta_{2}$-microglobulin, and yeast prions that couples primary nucleation and secondary nucleation leading the formation of additional nuclei from the end of preexisting filaments (called fragmentation) with linear growth. ${ }^{55}$ Moreover, Andersen et al. have demonstrated that new fibrils of glucagon could be formed from preexisting ones by branching. ${ }^{56}$ In addition, the kinetic signature of sigmoidal reaction showing the long lag phase followed by a rapid growth always indicates the generation of new aggregates during the aggregation of tau, ${ }^{25}$ islet, ${ }^{26,27}$ and insulin ${ }^{30}$ fibrils. The nucleation reaction orders extracted from the kinetic curves allow us to distinguish the fibril fragmentation and surfacecatalyzed nucleation. ${ }^{24}$

It is important to study the secondary nucleation because it might also contribute to the proliferation of fibrils in the brains of patients with Alzheimer's disease, thereby leading to its progression. Similar to our observations, several studies have shown that $A \beta 40$ fibril grows unidirectionally from the end of preexisting fibrils and the occurrence of secondary nucleation and fibril branching during fibril formation A $440,{ }^{15,56-58}$ IAPP, ${ }^{26}$ and other amyloid forming proteins using solution measurements and imaging techniques (e.g., AFM or total internal reflection fluorescence microscopy). ${ }^{15,56-58}$

However, the occurrence of secondary-nucleation sites on preexisting fibrils has not been hitherto demonstrated during fibrillogenesis from $A \beta 42$. We therefore sought to investigate this process and to elucidate the molecular factors governing its occurrence and specificity. Previous studies by Andersen et al..$^{20,56}$ and others ${ }^{59-61}$ demonstrated that parts of fibril surfaces have the capacity to nucleate fibrillization resulting in the branching, growth away or along the parent fibril. Under conditions that favored the formation of other fibril varieties (e.g., type-4 and type-5), no secondary-nucleation events were observed, even after the addition of fresh $A \beta 42-M$ (Fig. 4). On the basis of AFM observations combined with statistical analysis on fibrils and kinetic data, we conclude that inherent secondary-nucleation sites do indeed exist and that they are fibril-type specific. In the case of $A \beta 42$, only type-2 fibrils appear to be capable of growing via secondary-nucleation events.
We note that kinetic studies have already demonstrated their ability to incorporate primary and secondary nucleation pathways into the basic kinetic equations. However, no explicit attempts were made to describe a secondary nucleation pathway in $A \beta 42$ fibrillogenesis. Correlating the morphological changes observed by AFM during aggregation with the kinetic data combined with the systematic analysis of fibril height, periodicity, and height difference from the AFM data allowed us to provide a framework for the existence of secondary nucleation along fibrils and propose a testable assembly model for A $\mathrm{\beta} 42$ fibrillogenesis. Our findings affordfor the first time-evidences consistent with the secondary nucleation model that occurs upon the surfaces of preexisting fibrils, mainly on those of the type-2 variety.

\section{The concentration of monomeric $A \beta 42$ governs the assemblage of oligomers and protofibrils and the structure of the resulting fibrils}

Previous studies of our own ${ }^{33,34}$ and of other investigators ${ }^{62,63}$ indicate that the concentration of monomeric $A \beta$ (AB40 and $A \beta 42)$ governs the aggregation, toxicity, and pathology of these peptides. In the present study, we addressed the role of $A \beta 42-M$ in regulating the formation of fibrils at different stages of the process by means of realtime in situ AFM. Our results demonstrate that the presence of monomeric $A \beta 42$ is crucial for the assemblage of oligomers and protofibrils and for the growth and formation of fibrils. In the presence of A $342-\mathrm{M}$, spherical oligomers grew at a rate of $9.9 \pm$ $2.64 \mathrm{~nm} / \mathrm{min}$ to form elongated, smooth-contoured structures with an average length of $150-400 \mathrm{~nm}$. The average cross-sectional diameter of preformed oligomers that had been deposited on mica accorded well with that of dimers formed by hydrogen bonding between two $A \beta 42$ monomers. ${ }^{22}$ After their assemblage, the cross-sectional diameter of these oligomers remained unchanged. Upon the addition of fresh $A \beta 42-M$, we observed the sequential deposition of spherical oligomers upon their surfaces, giving rise eventually to structures with a cross-sectional diameter $(\sim 4 \mathrm{~nm})$ that was twice as great as that of the original oligomers. The final structure and cross-sectional diameter of the protofibrils corresponded to those of protofibrils observed in the ex situ samples (Fig. 1b). These findings demonstrate that preexisting oligomers serve as primary-nucleation sites for $A \beta 42$ fibrillogenesis. In the presence of low concentrations of $A \beta 42-M$, the spherical oligomers grew at a rate of only $0.75 \pm$ $0.25 \mathrm{~nm} / \mathrm{min}$ to form mainly larger spherical structures. This finding indicates that the presence of excess monomeric $A \beta 42$ is necessary to mediate the assemblage of spherical oligomers and the 
formation of protofibrils. Gosal et al. suggested the bifurcated pathway of $\beta_{2}$-microglobulin at which semiflexible (worm-like) fibrils were formed rapidly during assembly via a non-nucleated pathway that is distinct from the formation of relatively rigid longstraight fibrils. ${ }^{64}$ However, we could rule out this possible bifurcated pathway because we observed that protofibrils (height $=3.95 \pm 0.64 \mathrm{~nm}$ ) were formed by direct association of spherical oligomers and that these oligomers disappeared upon protofibril or fibril formation. However, we cannot rule out that other population the oligomers that form early represent off-pathway oligomers and re-enter the pathway of amyloid formation after monomer depletion. Our observations that monomeric $A \beta 42$ yields predominantly type-1 and type-2 fibrils, whereas mixtures of $A \beta 42-M$ and $A \beta 42-P F$ give rise mainly to type- 4 and type- 5 fibrils, likewise demonstrate that the concentration of free monomers is important for the growth and association of fibrils.

The molecular mechanisms underlying $A \beta$ fibrillogenesis and its role in the process of neurodegeneration are unknown. Progress in this field has been hampered by the heterogeneous size and morphology of the $A \beta$ aggregates and by our lack to comprehend the relationship between the different species. The studies presented provide novel insights into the mechanism of $A \beta 42$ fibril formation and growth, as well as into the molecular basis of polymorphism. In addition, we provide for the first time evidences for the existence of secondary-nucleation sites on the surfaces of $A \beta 42$ fibrils and demonstrate that $A \beta 42$ monomers play a crucial role in determining the structural properties and heterogeneity of the fibrils formed. The proposed mechanistic model indicates that a lowering of the levels of $A \beta$ peptides and a targeting of their growth and secondary nucleation represent viable therapeutic options for the inhibition of amyloid formation and the progression of neurodegeneration in Alzheimer's disease and related disorders.

\section{Materials and Methods}

\section{Preparation of monomeric and protofibrillar forms of A $\beta 42$}

As described in Jan et al., $1 \mathrm{mg}$ of lyophilized A 42 (Yale University, New Haven, CT, USA) was dissolved in $50 \mu$ of pure dimethyl sulfoxide (Sigma-Aldrich). ${ }^{52}$ We then added $800 \mu \mathrm{l}$ of ultra-pure water and $10 \mu \mathrm{l}$ of $2 \mathrm{M}$ Tris-base (pH 7.6) (Sigma-Aldrich) to the mixture, which was centrifugated at $600 \mathrm{~g}$ for $4 \mathrm{~min}$ at $4{ }^{\circ} \mathrm{C}$. The supernatant was aspirated and applied to a size-exclusion column (SEC; Superdex 75, GE Healthcare, Switzerland), which had been pre-equilibrated with $10 \mathrm{mM}$ Tris- $\mathrm{HCl}(\mathrm{pH}$ 7.4). Two $A \beta 42$ fractions - consisting of monomers (A $342-M)$ and protofibrils (Aß42-PF) -were separately collected at different elution times according to their molecular weight characteristics. Their absorbances were measured at a wavelength of $280 \mathrm{~nm}$ in an ultraviolet spectrometer (Varian). The concentration of the $A \beta 42$ species in each fraction was determined using a molar extinction coefficient of $1490 \mathrm{M}^{-1} \mathrm{~cm}^{-1}$ and adjusted to a final value of $20 \mu \mathrm{M}$. These solutions were used in our fibrillogenesis study.

\section{Preparation of $\mathrm{A} \beta 42$ fibrils}

The SEC-purified fractions of $A \beta 42-M$ and $A \beta 42-P F$ and a mixture of the two species were incubated separately for 1 month at $37{ }^{\circ} \mathrm{C}$ without agitation. To monitor the growth of fibrils, we added a freshly purified fraction of $A \beta 42-M$ $(20 \mu \mathrm{M})$ to a tube containing the preformed fibrils. The morphology of the fibrils was monitored at regular time intervals by TEM and AFM. The kinetics and extent of fibril formation were assessed using the ThT-binding assay.

\section{ThT-binding assay}

A 70- $\mu$ l aliquot of each $A \beta 42$ sample was added to a tube containing $20 \mu \mathrm{l}$ of $100 \mu \mathrm{M}$ ThT and $10 \mu \mathrm{l}$ of $500 \mathrm{mM}$ glycine- $\mathrm{NaOH}(\mathrm{pH} 8.5)$ (Sigma-Aldrich). The resulting solution $(100 \mu \mathrm{l})$ was then transferred to a 384-well fluorescence plate (Nunc), which was scanned with an Analyst AD fluorometer (Molecular Devices, Switzerland). Fluorescence was excited at a wavelength of $450 \mathrm{~nm}$, and its emission measured at one of $485 \mathrm{~nm}$.

\section{Sample preparation for ex situ AFM}

A substrate of freshly cleaved mica was incubated with a $10-\mu l$ drop of $0.05 \%$ (v/v) APTES (Fluka) in Milli-Q water for $1 \mathrm{~min}$ at ambient temperature. After rinsing with Milli-Q water, we dried the APTES-modified mica by the passage of a gentle flow of gaseous nitrogen. At defined time intervals, a $10-\mu$ l aliquot of each $A \beta 42$ sample was applied to the APTES-modified mica. After an equilibration period of $10 \mathrm{~min}$, the substrate was rinsed with Milli-Q water and then dried by the passage of gaseous nitrogen. AFM was performed in a Nanoscope IIla (Santa Barbara, CA), which was operated in the tapping mode under ambient conditions using a rectangular cantilever of silicon (Veeco Probes). The resonance frequency of the probe ranged between 96 and $168 \mathrm{kHz}$, and the spring constant was approximately $5 \mathrm{~N} / \mathrm{m}$.

\section{Sample preparation for in situ AFM}

A 5- $\mu$ l drop of a 1- $\mu \mathrm{M}$ solution of $A \beta 42-M$, which had been incubated for $4 \mathrm{~h}$, was applied to the substrate of APTESmodified mica. After an equilibration period of $30 \mathrm{~s}$, the mica was rinsed with Milli- $Q$ water to remove non-adherent $A \beta 42$. Once a stable image of oligomeric $A \beta 42$ was acquired on the substrate, $30 \mu \mathrm{l}$ of a $3-\mu \mathrm{M}$ solution of $A \beta 42-M$, oligomeric $A \beta 42$, or $A \beta 42-P F$ was injected into the fluid cell. The substrate was continuously monitored for up to $7 \mathrm{~h}$. When no further growth was observed, $30 \mu \mathrm{l}$ of a 3- $\mu \mathrm{M}$ solution of $\mathrm{A} \beta 42-\mathrm{M}$ was injected into the fluid cell to observe the growth of the elongated oligomers and the formation of protofibrils. AFM was performed in the tapping 
mode using a fluid cell that contained $10 \mathrm{mM}$ Tris- $\mathrm{HCl}$ buffer and a triangular cantilever of silicon nitride (Bruker Probes). The resonance frequency of the probe ranged between 9 and $10 \mathrm{kHz}$, and the spring constant was approximately $0.08 \mathrm{~N} / \mathrm{m}$. AFM images were flattened using the built-in software; background noise, brightness, and contrast were suitably adjusted. The statistical analysis of the images was conducted using Nanoscope Illa software.

\section{Acknowledgements}

This work is supported by the National Competence Center in Biomedical Imaging.

Author Contributions. J.S.J. performed AFM imaging, analyzed the data, and wrote the paper. A.A. prepared SEC-purified A 442 samples, performed electron microscopy imaging, and measured ThT assay. R.M. wrote the paper. H.A.L. and G.D. designed the research, analyzed the data, and wrote the paper. All authors contributed to the discussion of results and commented on the manuscript.

Competing Financial Interests. The authors declare no competing financial interests.

\section{Supplementary Data}

Supplementary data to this article can be found online at http://dx.doi.org/10.1016/j.jmb.2013.02.005

Received 1 October 2012;

Received in revised form 1 February 2013;

Accepted 5 February 2013

Available online 13 February 2013

$$
\begin{array}{r}
\text { Keywords: } \\
\text { amyloid; } \\
\text { mechanism; } \\
\text { nucleation; } \\
\text { atomic force microscopy; } \\
\mathrm{A} \beta 42
\end{array}
$$

\begin{abstract}
Abbreviations used:
AFM, atomic force microscopy; TEM, transmission electron microscopy; $A \beta$, amyloid $\beta$; APTES,

3-aminopropyl triethoxysilane; ThT, thioflavin $\mathrm{T}$; SEC, size-exclusion column.
\end{abstract}

\section{References}

1. Glenner, G. G. \& Wong, C. W. (1984). Alzheimer's disease: initial report of the purification and charac- terization of a novel cerebrovascular amyloid protein. Biochem. Biophys. Res. Commun. 120, 885-890.

2. Masters, C. L., Simms, G., Weinman, N. A., Multhaup, G., McDonald, B. L. \& Beyreuther, K. (1985). Amyloid plaque core protein in Alzheimer disease and Down syndrome. Proc. Natl Acad. Sci. USA, 82, 4245-4249.

3. Busciglio, J., Gabuzda, D. H., Matsudaira, P. \& Yankner, B. A. (1993). Generation of $\beta$-amyloid in the secretory pathway in neuronal and nonneuronal cells. Proc. Natl Acad. Sci. USA, 90, 2092-2096.

4. Shoji, M., Golde, T. E., Ghiso, J., Cheung, T. T., Estus, S., Shaffer, L. M. et al. (1992). Production of the Alzheimer amyloid $\beta$ protein by normal proteolytic processing. Science, 258, 126-129.

5. Iwatsubo, T., Odaka, A., Suzuki, N., Mizusawa, H., Nukina, N. \& Ihara, Y. (1994). Visualization of $A \beta 42(43)$ and $A \beta 40$ in senile plaques with end-specific $A \beta$ monoclonals: evidence that an initially deposited species is $A \beta 42(43)$. Neuron, 13, 45-53.

6. Merz, P. A., Wisniewski, H. M., Somerville, R. A., Bobin, S. A., Masters, C. L. \& Iqbal, K. (1983). Ultrastructural morphology of amyloid fibrils from neuritic and amyloid plaques. Acta Neuropathol. 60, 113-124.

7. Terry, R. D., Gonatas, N. K. \& Weiss, M. (1964). Ultrastructural studies in Alzheimer's presenile dementia. Am. J. Pathol. 44, 269-297.

8. Roher, A., Wolfe, D., Palutke, M. \& Kukuruga, D. (1986). Purification, ultrastructure, and chemical analysis of Alzheimer disease amyloid plaque core protein. Proc. Natl Acad. Sci. USA, 83, 2662-2666.

9. Andreu, J. M. \& Timasheff, S. N. (1986). The measurement of cooperative protein self-assembly by turbidity and other techniques. Methods Enzymol. 130, 47-59.

10. Jarrett, J. T. \& Lansbury, P. T., Jr. (1993). Seeding "one-dimensional crystallization" of amyloid: a pathogenic mechanism in Alzheimer's disease and scrapie? Cell, 73, 1055-1058.

11. Walsh, D. M., Lomakin, A., Benedek, G. B. \& Condron, M. M. (1997). Amyloid $\beta$-protein fibrillogenesis: detection of a protofibrillar intermediate. J. Biol. Chem. 272, 22364-22372.

12. Serpell, L. C. (2000). Alzheimer's amyloid fibrils: structure and assembly. Biochim. Biophys. Acta, 1502, 16-30.

13. Komatsu, H., Link, E. F., Sharp, K. A., Rastogi, T. \& Axelsen, P. H. (2010). Intrinsic linear heterogeneity of amyloid $\beta$ protein fibrils revealed by higher resolution mass-per-length determinations. J. Biol. Chem. 285, 41843-41851.

14. Bauer, H. H., Aebi, U., Häner, M., Hermann, R. \& Müller, M. (1995). Architecture and polymorphism of fibrillar supramolecular assemblies produced by in vitro aggregation of human calcitonin. J. Struct. Biol. 115, 1-15.

15. Yagi, H., Ban, T., Morigaki, K., Naiki, H. \& Goto, Y. (2007). Visualization and classification of amyloid $\beta$ supramolecular assemblies. Biochemistry, 46, 15009-15017.

16. Kodali, R., Williams, A. D., Chemuru, S. \& Wetzel, R. (2010). $A \beta(1-40)$ forms five distinct amyloid structures whose $\beta$-sheet contents and fibril stabilities are correlated. J. Mol. Biol. 20, 503-517. 
17. Goldsbury, C., Frey, P., Olivieri, V., Aebi, U. \& Müller, S. A. (2005). Multiple assembly pathways underlie amyloid- $\beta$ fibril polymorphisms. J. Mol. Biol. 352 , 282-298.

18. Paravastu, A. K., Qahwash, I., Leapman, R. D., Meredith, S. C. \& Tycko, R. (2009). Seeded growth of $\beta$-amyloid fibrils from Alzheimer's brain-derived fibrils produces a distinct fibril structure. Proc. Natl Acad. Sci. USA, 106, 7443-7448.

19. Shirahama, T. \& Cohen, A. S. (1967). High-resolution electron microscopic analysis of the amyloid fibril. J. Cell Biol. 33, 679-708.

20. Andersen, C. B., Otzen, D., Christiansen, G. \& Rischel, C. (2007). Glucagon amyloid-like fibril morphology is selected via morphology-dependent growth inhibition. Biochemistry, 46, 7314-7324.

21. Kowalewski, T. \& Holtzman, D. M. (1999). In situ atomic force microscopy study of Alzheimer's $\beta$ amyloid peptide on different substrates: new insights into mechanism of $\beta$-sheet formation. Proc. Natl Acad. Sci. USA, 96, 3688-3693.

22. Mastrangelo, I. A., Ahmed, M., Sato, T., Liu, W., Wang, C., Hough, P. \& Smith, S. O. (2006). Highresolution atomic force microscopy of soluble $A \beta 42$ oligomers. J. Mol. Biol. 358, 106-119.

23. Harper, J. D., Wong, S. S., Lieber, C. M. \& Lansbury, P. T., Jr. (1997). Observation of metastable $A \beta$ amyloid protofibrils by atomic force microscopy. Chem. Biol. 4, 119-125.

24. Cohen, S. I. A., Vendruscolo, M., Dobson, C. M. \& Knowles, T. P. J. (2012). From macroscopic measurements to microscopic mechanisms of protein aggregation. J. Mol. Biol. 421, 160-171.

25. Ramachandran, G. \& Udgaonkar, J. B. (2012). Evidence for the existence of a secondary pathway for fibril growth during the aggregation of tau. J. Mol. Biol. 421, 296-314.

26. Padrick, S. B. \& Miranker, A. D. (2002). Islet amyloid: phase partitioning and secondary nucleation are central to the mechanism of fibrillogenesis. Biochemistry, 41, 4694-4703.

27. Ruschak, A. M. \& Miranker, A. D. (2007). Fiberdependent amyloid formation as catalysis of an existing reaction pathway. Proc. Natl Acad. Sci. USA, 104, 8691-8696.

28. Ferrone, F. A., Hofrichter, J., Sunshine, H. R. \& Eaton, W. A. (1980). Kinetic studies on photolysis-induced gelation of sickle cell hemoglobin suggest a new mechanism. Biophys. J. 32, 361-380.

29. Collins, S. R., Douglass, A., Vale, R. D. \& Weissman, J. S. (2004). Mechanism of prion propagation: amyloid growth occurs by monomer addition. PLOS Biol. 2, 1582-1590.

30. Nielsen, L., Khurana, R., Coats, A., Frokjaer, S., Brange, J., Vyas, S. et al. (2001). Effect of environmentral factors on the kinetics of insulin fibril formation: elucidation of the molecular mechanism. Biochemistry, 40, 6036-6046.

31. Hofrichter, J., Ross, P. D. \& Eaton, W. A. (1974). Kinetics and mechanism of deoxyhemoglobin $s$ gelation: a new approach to understanding sickle cell disease. Proc. Natl Acad. Sci. USA, 71, 4864-4868.

32. Bishop, M. F. \& Ferrone, F. A. (1984). Kinetics of nucleation-controlled polymerization. A perturbation treatment for use with a secondary pathway. Biophys. J. 46, 631-644.

33. Jan, A., Gokce, O., Luthi-Carter, R. \& Lashuel, H. A. (2008). The ratio of monomeric to aggregated forms of $A \beta 40$ and $A \beta 42$ is an important determinant of amyloid- $\beta$ aggregation, fibrillogenesis, and toxicity. J. Biol. Chem. 283, 28176-28189.

34. Jan, A., Adolfsson, O., Allaman, I., Buccarello, A. L., Magistretti, P. J., Pfeifer, A. et al. (2011). A 442 neurotoxicity is mediated by ongoing nucleated polymerization process rather than by discrete $A \beta 42$. J. Biol. Chem. 286, 8585-8596.

35. Lührs, T., Ritter, C., Adrian, M., Riek-Loher, D., Bohrmann, B., Döbeli, H. et al. (2005). 3D structure of Alzheimer's amyloid- $\beta(1-42)$ fibrils. Proc. Natl Acad. Sci. USA, 102, 17342-17347.

36. Adamcik, J., Jung, J. M., Flakowski, J., Rios, P. D. L., Dietler, G. \& Mezzenga, R. (2010). Understanding amyloid aggregation by statistical analysis of atomic force microscopy images. Nat. Nanotechnol. 5, 423-428.

37. Karsai, A., Grama, L., Murvai, Ü., Soós, K., Penke, B. \& Kellermayer, M. S. Z. (2007). Potassium-dependent oriented growth of amyloid $\beta 25-35$ fibrils on mica. Nanotechnology, 18, 345102.

38. Petkova, A. T., Leapman, R. D., Guo, Z., Yau, W. M., Mattson, M. P. \& Tycko, R. (2005). Self-propagating, molecular-level polymorphism in Alzheimer's $\beta$-amyloid fibrils. Science, 307, 262-265.

39. Paravastu, A. K., Petkova, A. T. \& Tycko, R. (2006). Polymorphic fibril formation by residues 10-40 of the Alzheimer's $\beta$-amyloid peptide. Biophys. J. 90, 4618-4629.

40. Goldsbury, C. S., Wirts, S., Müller, S. A., Sunderji, S., Wicki, P., Aebi, U. \& Frey, P. (2000). Studies on the in vitro assembly of $A \beta 1-40$ : implications for the search for $A \beta$ fibril formation inhibitors. J. Struct. Biol. 130, 217-231.

41. Goldsbury, C. S., Cooper, G. J., Goldie, K. N., Müller, S. A., Saafi, E. L., Gruijters, W. T. et al. (1997). Polymorphic fibrillar assembly of human amylin. J. Struct. Biol. 119, 17-27.

42. Jiménez, J. L., Nettleton, E. J., Bouchard, M., Robinsson, C. V., Dobson, C. M. \& Saibil, H. R. (2002). The protofilament structure of insulin amyloid fibrils. Proc. Natl Acad. Sci. USA, 99, 9196-9201.

43. Bessen, R. A. \& Marsh, R. F. (1992). Biochemical and physical properties of the prion protein from two strains of the transmissible mink encephalopathy agent. J. Virol. 66, 2096-2101.

44. Safar, J., Wille, H., Itri, V., Groth, D., Serban, H., Torchia, M. et al. (1998). Eight prion strains have $\mathrm{PrP}^{\mathrm{Sc}}$ molecules with different conformations. Nat. Med. 4, 1157-1165.

45. Chien, P. \& Weissman, J. S. (2001). Conformational diversity in a yeast prion dictates its seeding specificity. Nature, 410, 223-227.

46. Tanaka, M., Chien, P., Naber, N., Cooke, R. \& Weissman, J. S. (2004). Conformational variations in an infectious protein determine prion strain differences. Nature, 428, 323-328.

47. Goldsbury, C., Kistler, J., Aebi, U., Arvinte, T. \& Cooper, G. J. (1999). Watching amyloid fibrils grow by time-lapse atomic force microscopy. J. Mol. Biol. 285, 33-39. 
48. Parbhu, A., Lin, H., Thimm, J. \& Lal, R. (2002). Imaging real-time aggregation of amyloid $\beta$ protein (1-42) by atomic force microscopy. Peptides, 23 1265-1270.

49. Blackley, H. K. L., Sanders, G. H. W., Davies, M. C., Roberts, C. J., Tendler, S. J. B. \& Wilkinson, M. J. (2000). In-situ atomic force microscopy study of ß-amyloid fibrillization. J. Mol. Biol. 298, 833-840.

50. Arimon, M., Pérez, I. D., Kogan, M. J., Durany, N., Giralt, E., Sanz, F. \& Busquets, X. F. (2005). Fine structure study of $A \beta_{1-42}$ fibrillogenesis with atomic force microscopy. FASEB J. 19, 1344-1346.

51. Bartolini, M., Naldi, M., Fiori, J., Valle, F., Biscarini, F., Nicolau, D. V. \& Andrisano, V. (2011). Kinetic characterization of amyloid- $\beta$ 1-42 aggregation with a multimethodological approach. Anal. Biochem. 414, 215-225.

52. Jan, A., Hartley, D. M. \& Lashuel, H. A. (2010). Preparation and characterization of toxic $A \beta$ aggregates for structural and functional studies in Alzheimer's disease research. Nat. Protoc. 5, 1186-1209.

53. Bitan, G., Kirkitadze, M. D., Lomakin, A., Vollers, S., Benedek, G. \& Teplow, D. B. (2003). Amyloid $\beta$-protein assembly: $A \beta 40$ and $A \beta 42$ oligomerize through distinct pathways. Proc. Natl Acad. Sci. USA, 100, 300-335.

54. Heldt, C. L., Zhang, S. \& Belfort, G. (2010). Asymmetric amyloid fibril elongation: a new perspective on a symmetric world. Proteins, 79, 92-98.

55. Knowles, T. P. J., Waudby, C. A., Devlin, G. L., Cohen, S. I. A., Aguzzi, A., Vendruscolo, M. et al. (2009). An analytical solution to the kinetics of breakable filament assembly. Science, 326, 1533-1537.

56. Andersen, C. B., Yagi, H., Manno, M., Martorana, V., Ban, T., Christiansen, G. et al. (2009). Branching in amyloid fibril growth. Biophys. J. 96, 1529-1536.
57. Ban, T., Hamada, D., Hasegawa, K., Naiki, H. \& Goto, Y. (2003). Direct observation of amyloid fibril growth monitored by thioflavin T fluorescence. J. Biol. Chem. 278, 16462-16465.

58. Ban, T., Hoshino, M., Takahashi, S., Hamada, D., Hasegawa, K., Naiki, H. \& Goto, Y. (2004). Direct observation of $A \beta$ amyloid fibril growth and inhibition. J. Mol. Biol. 344, 757-767.

59. Jansen, R., Dzwolak, W. \& Winter, R. (2005) Amyloidogenic self assembly of insulin aggregates probed by high resolution atomic force microscopy. Biophys. J. 88, 345102.

60. Anderson, M., Bocharova, O. V., Makarava, N., Breydo, L., Salnikov, V. V. \& Baskakov, I. V. (2006). Polymorphism and ultrastructural organization of prion protein amyloid fibrils: an insight from high resolution atomic force microscopy. J. Mol. Biol. 358, 580-596.

61. Rogers, S. S., Krebs, M. R. H., Bromley, E. H. C., van der Linden, E. \& Donald, A. M. (2006). Optical microscopy of growing insulin amyloid spherulites on surfaces in vitro. Biophys. J. 90, 1043-1054.

62. Kanemitsu, H., Tomiyama, T. \& Mori, H. (2003). Human neprilysin is capable of degrading amyloid $\beta$ peptide not only in the monomeric form but also the pathological oligomeric form. Neurosci. Lett. 350, 113-116.

63. Kuperstein, I., Broersen, K., Benilova, I., Rozenski, J., Jonckheere, W., Debulpaep, M. et al. (2010). Neurotoxicity of Alzheimer's disease $A \beta$ peptides is induced by small changes in the $A \beta_{42}$ to $A \beta_{40}$ ratio. EMBO J. 29, 3408-3420.

64. Gosal, W. S., Morten, I. J., Hewitt, E. W., Smith, A., Thomson, N. H. \& Radford, S. E. (2005). Competing pathways determine fibril morphology in the selfassembly of $\beta_{2}$-microglobulin into amyloid. J. Mol. Biol. 351, 850-864. 